\title{
A review on multiscale modelling and simulation for polymer nanocomposites
}

\author{
Annu Sharma ${ }^{1, *}$, Mamta Devi ${ }^{2}$ \\ ${ }^{1}$ Thakur PG College of Education, Dhaliara, Himachal Pradesh - 177103 India \\ ${ }^{2}$ Energy Research Centre, Panjab University, Chandigarh-160014, India.
}

\begin{abstract}
The present paper is focused on understanding multiscale modelling to obtain a bridge among different time and length scales of simulation techniques. These techniques are vital as it holds the potential to understand and predict the capabilities of polymer nanocomposites (PNCs). However, an appropriate approach in controlling the interfacial interaction between nanoparticle and polymer in nanocomposites structure is still needed to develop. In this review, an initial brief introduction to various trending simulation techniques has been discussed at all three levels of scale (nm, $\mu \mathrm{m}, \mathrm{mm})$. Later, descriptive study on fundamental issues such as thermodynamics, kinetics, mechanical properties, and morphology has been studied deeply. The multiscale modelling bridges the gaps of simulation between the different scales of models from molecular to mesoscale levels working over the broad range of length and timescale. Through the sequential, adaptive, and concurrent approaches, we can develop a system that may comprehend multiscale modelling adaptive resolution approach has recently added approach the molecule of the subject can shift their position freely in the domain and through this approach and studied the Brownian motion. Co-The co-current coach is also termed as handshaking path and it is linked aiming at different scale models. Covering the rigid techniques smoothly and linking them at different scales helps in normalizing the statistical behaviour.
\end{abstract}

Keywords: Polymer nanocomposite; Multiscale modelling; Nanostructure; Nanoparticle; Interface; Simulation;

\section{Introduction}

Recent studies have shown remarkable attention towards the Polymer Nanocomposites at both the scientific and industrial levels. Reinforced material has become the prior interest of researchers as it provides reliable application over a wide range in society. The 2-D structure nano-filler forms nanocomposites that would be heterogeneities in the nanometre range [1-3] Nanocomposite usually covers the range between inorganic glasses and organic polymers [4] Nano Fillers reinforcements in polymers have enhanced their performance in the long term perspective. The present paper would provide a brief critical review of the literature and some of the results [5] Scientific and technical literature has reported the improvement of properties in polymer nanocomposites compared to pristine polymers. This statement refers to an improvement of polymer properties from the standpoint of polymer application [1,3-10]. Experimental manipulation during fabrication exhibits unstable resultant behaviour. Moreover, the experimental approach can't cover all the aspects in one-way testing. Therefore, simulation and modelling technique becomes very important, as these tools have a higher degree of accessibility and accuracy(fig.1). They can study the possibilities at larger lengths and time scales. Also guiding in designing the desired hierarchical strategy to be followed up for the experimental procedure. 


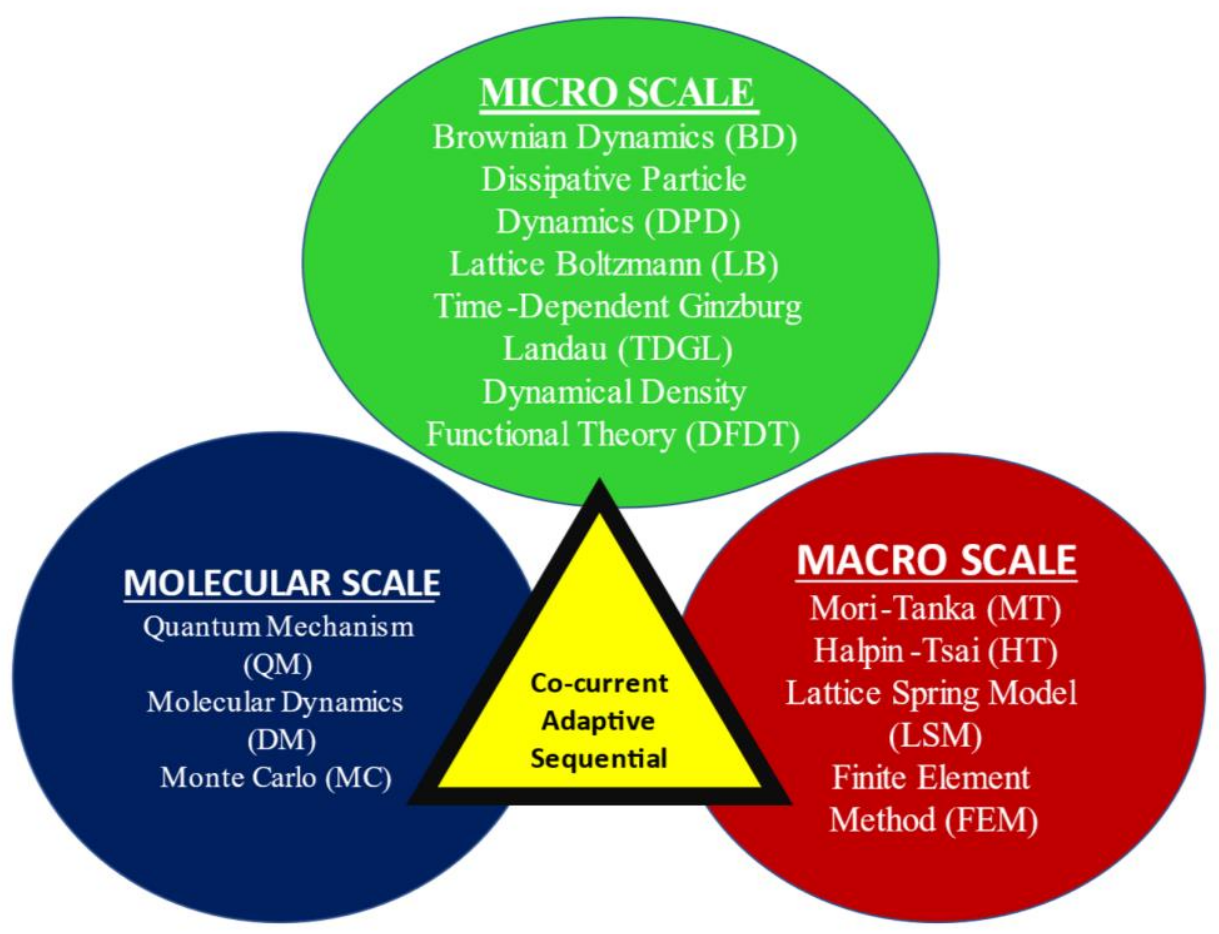

Fig.1. Representation route of interlink among different length scales of simulation and modelling techniques.

\section{Polymer nanocomposites}

Since Toyota Group has done a successful experiment over the nanocomposites, it has become an impressive and viable approach to industrial applications [11,12] They were able to polymerize their $\varepsilon$-caprolactam with the addition of organoclay in the interlayer matrix of clay to produce a Nylon 6-clay hybrid. At a specific loading of only $4.2 \mathrm{wt} \%$, they doubled the tensile strength up to $\sim 50 \%$, and Heat distortion temperature (HDT) increased up to $80^{\circ} \mathrm{C}$ in comparison to the pristine polymer. This astonishing result encouraged many researchers to work on the significant enhancement of pristine polymer properties upon addition of such minute loading of organoclay. Reinforcement at nanoscale integrates the properties of polymer with the minuscule count of Nanofiller. Modified Ceramic nanofiller has become an attractive part of the interest in the coatings field upon its best compatibility with the Polyurethane as nanofiller for the processing nanocomposite[7,13-15]. They not even have improved the chemical interfaces between the cross-linkages of the nanocomposites but have supported it with its physical appearances too. Different Processing techniques quench the bonds differently, which results in an improvement in different sites of PU[14] It requires more attention of researchers to evident the changes developed at interfaces and investigation through highly focused magnifying equipment.

Indeed, experimental results may characterize it but still, it's a challenge to manipulate its synthesis due to lack of exact characterization over its structure. Fabricating such material is seems to be an empirical problem and keen control over their properties is far to achieve. Therefore, for designing desired material and guiding experimental work we need to take the lead from simulation or computer modelling. The main focus of this review article is to discuss the effects of a few processing parameters on the behaviour and properties of modified material through the application of simulation and computer modelling. Initially, the brief introduction to the ruling expressions, parameters and conditions of simulation techniques such as Monte Carlo, Molecular dynamics, CDM, etc along with the margins and drawbacks have 
been scrutinized. Three major categories have formed to distinguish between techniques based on the level of exploration, these are Molecular scale (molecular dynamic method, Monte Carlo), Micro scale (dissipative particle dynamics, density functionalized theory etc) and Mesoscale/Macro scale (Finite element method, etc)

The following issues can be resolved with this approach: To study the major two aspects of any synthesis i.e. its thermodynamics and kinetics of formation. However, characterization can be done at the macro to micro-level but here in this review, we focus in particular, to characterize the structure of the molecular structures and dynamics at the interface between nanoparticles and the polymer matrix. The Rheological behaviour attained by the polymer in addition to nanofiller as it would help in optimizing the processing conditions and the reinforcement mechanism of nanocomposites.

In this review, discussion over the application on modelling and simulation techniques have been done with covering the broad-scale range of methodologies to summarize the advancements that can use in the fundamental understanding of polymer nanocomposites at nanoscale use materials likewise nano-tubes, clay platelets, etc. The possible routes can be applied to interconnect the different length and time scale methods.

\section{Molecular scale modelling}

Modelling of polymer nanocomposite at its molecular-scale helps to predict its thermodynamics and kinetics of synthesis, its structure and interactions at the molecular level. This is widely practice method sub-categorised into two main simulation techniques MD and MC mechanisms.

\section{MD (molecular dynamics)}

is a technique that evaluates the time evaluation of interacting particles and structure[16] Its three major constitutes are a) input conditions, b) force of interaction and c) time-dependent changes in Newtonian equation of motion for the system and control over pressure and temperature along with a parent selection of boundary conditions leads to interpret meaningful prediction.

The equation of motion is givthe en by

$F_{i}(t)=m_{i} \frac{d^{2} r_{i}}{d t^{2}}$

where $F_{i} \rightarrow$ is a force acting on any $i^{\text {th }}$ atom/ particle

$m_{i}$ is the atomic mass

$r_{i} \rightarrow$ is the atomic position

The Criteria chosen for selecting an accurate and reliable force field depend upon the bonded and non-bonded force fields calculation;

$$
\begin{aligned}
\mathrm{U}\left(r_{1} \rightarrow, r_{2}, \ldots \ldots \ldots \ldots r_{N}=\sum_{i_{\text {bond }}}^{N_{\text {bond }}} U_{\text {bond }}\left(i_{\text {bond }}, r_{a}, r_{b}\right)\right. & \\
& +\sum_{i_{\text {angle }}}^{N_{\text {angle }}} U_{\text {angle }}\left(i_{\text {angle }}, r_{a}, r_{b}, r_{c}\right) \\
& +\sum_{\text {torsion }}^{N_{\text {torsion }}} U_{\text {torsion }}\left(i_{\text {torsion }}, r_{a}, r_{b}, r_{c}, r_{d}\right)
\end{aligned}
$$




$$
\begin{gathered}
+\sum_{i_{\text {inversion }}}^{N_{\text {inversion }}} U_{\text {inversion }}\left(r_{a}, r_{b}, r_{c}, r_{d}\right) \\
+\sum_{i=1}^{N-1} \sum_{j>1}^{N} U_{\text {vanderwaal }}\left(i, j, r_{a}, r_{b}\right)+\sum_{i=1}^{N-1} \sum_{j>1}^{N} U_{\text {electrostatic }}\left(i, j, r_{a}, r_{b}\right)
\end{gathered}
$$

where, bond, angle, torsion and inversion are bonded interactions. Whereas the other two are non-bonded interactions of van der Waals energy and electrostatic forces.

$r \rightarrow$ is a position vector of atom/particle

$\mathrm{N}$ is a no. of interaction

\section{I bond is a bonded interaction}

$i, j$ is non-bonded interaction (can be of two types van der Waals and electrostatic forces) MD simulation technique allows investigating the effect of nanofiller on nanocomposite matrix and its interface.

\section{Mc (Monte Carlo)}

It is a kind of flexible technique that works on randomly selected populations for the investigation of any desired property This simulation technique is also required to follow three steps a) producing a probabilistic model, b) Solving that model with a suitable numerical method and c) Analyzing data using statistical methods. MC mechanism can only provide the prediction equilibrium conditions whereas, MD tends to give the information about equilibrium as well as non-equilibrium conditions.

Generally, Molecular Dynamics simulation can be performed over various ensembles for example $\mu$ VT (grand canonical), NVE (microcanonical), NVT (canonical) and NPT(Isothermal-isobaric). Here NVT on the Hamiltonian mechanism in goes goes difference of position othe number goesi $\rightarrow \mathrm{j}$ predicts $\Delta$ another in thenumber theta acceptance of this operation that generates acceptedcomputationinhe . Certain probabilitysubscriptions given as;

$$
P_{i \rightarrow j} \alpha \exp \left(\frac{-\Delta H}{T k_{B}}\right) \text { where } k_{B} \text { is the Boltzmann constant. }
$$

If, $\theta \leq$ anotherin probability, accepted. Otherwise, a new configuration is not acceptedcomputationin repeats the process by choosing another target particle. Same as the MVT, $\mu$ VT works on replacement of one atom say $\mathrm{j}$ by other atom and it also accepts its move based on $\Delta \mathrm{U} \geq 0$ computatiothe nal condition is accepted further $P_{i \rightarrow j} \alpha \exp \left(\frac{-\Delta U}{T k_{B}}\right)$, the canonical method has the conditions for certain probability acceptance is similarly applied for grand canonical.

\section{Microscale modeling methods}

To develop the structural evolution of nanofiller - polymer covering the changes in its phase separation and the interaction among the nanoparticles and the polymer matrix. The simulation under this category follows the continuum method by explaining hydrodynamic behavioural of polymer nanocomposite whereas the atomistic method is critical and difficult to practice. For modeling, there are a few well-designed and described methods named as BD, DPD, LB, time-dependent Ginsburg-landau (TDGL) theory and Dynamics DFT. These methods track the phenomena on the length and time scale which is an advantage over the MD technique. 


\section{Brownian Dynamics}

$\mathrm{BD}$ can be termed as a random simulation considering the diffusion as the main content of interest. In addition to the characteristics of the MD technique, BD can work on a very minute time scale (in fact up to a few nanoseconds) which makes it more efficient for simulation of nanofiller polymer nanocomposites. BD system is a sub-division of micro scale methods, it manipulates the inputs based on a time scale Generally, used to study the relatiparenteen solvent-polymer where implicit continuum solvent description is been used. Its guiding equation is consisting of dissipative and random force terms whparentl the effect of removal of solvent from polymer and it is named as Langevin equation, which is given by:

$$
F_{i}(t)=\sum_{j \neq 1} F_{i j}^{C}-\gamma p_{i}+\sigma \zeta_{i}(t),
$$

where $F_{i j}^{C}$ is a conservative force of $\mathrm{j}$ particle on i particle

$\gamma, \sigma$ are constants

$p_{i}$ is a momentum apparently $\mathrm{i}$

$\zeta_{i}(t)$ is a Gaussian random noise term

Although BD provides the information about diffusion among the polymer and solvent it loses its track of the hydrodynamic behavior as it doesn't obey the Navier Stokes equation of motion. It's a consequence of not focusing on the conservation of the energy and momentum forces.

\section{DPD}

Covering to the limitations of the above techniques, Hoogerbruge and Koelman have developed the DPD technique. During the study of polymers, their flow patterns are a very crucial part. They have given the model that can obey the Navier stokes equation of motion and perform simulation on every possible polymer solution.

It is also a Particle-based model. DPD has also covered the importance of conservation along with dissipation and random (the targeting portions of $\mathrm{BD}$ ) terms in its model.

$$
\begin{gathered}
F_{i j}=F_{i j}^{C}+F_{i j}^{D}+F_{i j}^{R}, \\
F_{i j}^{C}=\prod_{0} \omega_{C}\left(r_{i j}\right) \hat{e}_{i j}, \\
F_{i j}^{D}=-\gamma \omega_{D}\left(r_{i j}\right)\left(\hat{e}_{i j} \cdot p_{i j}\right) \hat{e}_{i j}, \\
F_{i j}^{R}=\sigma \zeta_{i j} \omega_{R}\left(r_{i j}\right) s u b j
\end{gathered}
$$

whereas $r_{i j}=\left|r_{j}-r_{i}\right|, \hat{e}_{i j}=\frac{\hat{r}_{i j}}{r_{i j}}, \Pi_{0}$ is constant to fluid compressibility, $\gamma$ is friction coefficient, $\sigma$ is a noise amplitude and $\zeta \mathrm{ij}$ is random noise term and $\omega$ is weight function.

DPD technique simulates the interaction potential between polymer and nanofiller at the length and time scale. This makes it an effective technique. The conservative force decreases linearly with increasing its position among two particles.

\section{Lattice Boltzmann,}

Considering the lattice matrix is another technique named as LB investigating the lattice gas automation simulation by targeting at local particles velocity. The time scale is shortened into two-time steps for simulation i.e., Streaming 
(movement of particles to the nearest node) and collision (interaction among particles) as these effects change the magnitude and direction of particle velocity.LB surely treats the fluid dynamics of polymer solution with taking the help of the Boltzmann kinetic model or discrete velocity model. Equation which governs is as shown,

$$
\begin{aligned}
& n_{i}\left(x+e_{i}, t+1\right)=n_{i}(x, t)+\Omega_{i}(n(x, t)), i=0,1, \ldots . . N \\
& f_{i}^{\sigma}\left(x+e_{i} \Delta x, t+\Delta t\right)-f_{i}^{\sigma}(x, t)=\Omega_{i}\left(f_{i}^{\sigma}(x, t)\right), \sigma=1,2 \ldots \ldots S
\end{aligned}
$$

LB method can describe various aspects of the solution behaviour through in terms of free energy, thermodynamics, kinetics and hydrodynamics (flow patterns) etc. it is a conventional way of producing particle interaction into numerical modelling. But its limits in stabilizing the numerical results which makes it an unreliable modelling technique.

\section{TDGL}

TDGL method takes into consideration of simulation on polymer blends and copolymers, describing its structure at phase separation. This technique is capable of solving non-linear diffusion. TDGL suppress the free-energy function in the conversion of miscible to the immiscible region. Therefore, the model can be solved through this technique. These equations describe the motion of an incompressible fluid for two different segments

$\frac{\partial \phi(r, t)}{\partial t}=\nabla \cdot M \nabla \frac{\delta U[\phi(r, t)]}{\delta \phi(r, t)}+\zeta(r, t)$

Whereas, $\zeta(r, t)=$ thermal noise,

$\mathrm{U}=$ free energy,

$\mathrm{M}=$ mobility

$\phi=$ order parameter

$\frac{U[\phi(r)]}{k_{B} T}=\int d r$

$U_{F H}=$ Flory Huggins free energy defined as

$\frac{U_{F H}(\phi)}{k_{B} T}=\frac{\phi}{N_{A}} \ln \phi+\frac{(1-\phi)}{N_{B}} \ln (1-\phi)+\chi \phi(1-\phi)$

Where $\chi=$ enthalpic interaction between polymer components,

Critical values can also calculate wimoderniselp of Flory Huggins modeandse $\phi_{C} \wedge \chi_{C}$ in the above equation.

CDM (cell half-open method) have become simplified from TGDL to the form,

$\frac{U_{C D M}[\phi(r)]}{k_{B} T}=\int d r\left[-A \ln (\cosh \phi)+1 / 2(\phi)^{2}+\frac{D}{2} \vee \nabla \phi(r) \vee^{2}\right]$

Here, the isotropic term gets discretized instead of the Laplacian form. TGDL and CDM are widely practiced for phase separation in polymer nanocomposites. 
Improvement in the functioning of TGDL is developed in DFT, majorly the effect of Viscoelastic and free energy functions as it affects the integral path of polymer blends. For improving these functions Gaussian mean-free path and Flory Huggins's mobility is important. Following equations governing the simulation pattern;

$$
\frac{\partial \phi(r, t)}{\partial t}=M \nabla^{2} \frac{\delta U[\phi(r, t)]}{\delta \phi(r, t)}-\Gamma[\phi(r, t)-\lambda]+\xi(r, t)
$$

DFT approach is efficient in conformed the non-equilibrium density profile by considering the interaction potential also statistical thermodynamics.

$$
\text { Where } \phi=\rho_{A}-\rho_{B}, \quad \mathrm{U} \phi=U_{l}+U_{c p l}
$$

$$
\begin{aligned}
U_{l}=\int d r\left[f_{1} \phi((r, t))+\frac{D}{2}(\nabla \phi(r, t))^{2}\right], \\
U_{c p l}=C \int d r \sum V(r-R)\left(\phi(\mathrm{r}, \mathrm{t})-\phi_{s}\right)^{2}, \phi_{s}=1
\end{aligned}
$$

The value of $\mathrm{R}$ is obtained from the Langevin equation: $R_{i}=M_{P}\left(f_{i}-\frac{\partial U(\phi)}{\partial R_{i}}\right)+\eta_{i}$

Where $M_{P}=$ particle mobility

$$
\begin{aligned}
& f_{i}=\text { force of interaction } \\
& \eta_{i}=\text { Gaussian white noise }
\end{aligned}
$$

\section{Mesoscale and Macroscale Method}

To propose a model at the macro scale the investigation has to be surrounded at the macro level by avoiding digging at the atomic level. Averages forces and energies come into account in this simulation. Mesoscale illustrations follow some fundamental laws such as continuity (conservation of mass), equilibrium (Newton's second law), the moment of momentum principle (rate of change of angular momentum), conservation of energy (first law of thermodynamics) and conservation of entropy (second law of thermodynamics). these are the roots of the continuum model developed with the study of stress and strain over the time on the polymer nanocomposite.

\section{Micro mechanism}

Based on the continuum model assumptions that need to be considered are a) linear relation of elasticity between filler and polymer matrix, b) uniformity on the aspect ratio of the filler, c) best intercalation between polymer and filler.

Initially, elasticity need to study, the relation develop of stress versus infinitesimal strain is given as

For filler: $\quad \sigma^{\mathrm{f}}=\mathrm{C}^{\mathrm{f}} \mathcal{E}^{\mathrm{f}}$

For matrix: $\quad \sigma^{\mathrm{m}}=\mathrm{C}^{\mathrm{m}} \varepsilon^{\mathrm{m}}$,

C - Tensor stiffness

Then the averages come in the model in such a pattern followed by the system. Stress and strain effect on volume.

$\bar{\sigma}=\frac{1}{V} \int_{V(\text { initial })}^{V(\text { final })} \sigma(x) d V$

$\bar{\varepsilon}=\frac{1}{V} \int_{V(\text { initial })}^{V(\text { final })} \varepsilon(x) d V$

These equations can be written accordingly filler and matrix format for volume fraction.

The overall relationship between filler and the matrix can be written as;

$\bar{\sigma}=v_{f} \bar{\sigma}_{f}+v_{f} \overline{\bar{\sigma}}_{f}$ 
$\bar{\varepsilon}=v_{m} \bar{\varepsilon}_{m}+v_{m} \bar{\varepsilon}_{m}$

Calculating the average properties of the nanocomposites using stiffness parameter given as;

$\bar{\sigma}=C \bar{\varepsilon}$

Then, by using strain-concentration tensor $\mathrm{A}$ is obtained from a given equation;

$\mathrm{C}=C=C_{m}+v_{f}\left(C_{f}-C_{m}\right) A$.

\section{Halpin-Tsai Model}

This model works for the prediction of mechanical properties of the samples using young's modulus concept for calculation for unidirectional stiffness as a function of aspect ratio. Comprising of two parameters of young's modulus longitudinal and transverse give as $\mathrm{E}_{\mathrm{L}}$ and $\mathrm{E}_{\mathrm{T}}$

$\xi_{\mathrm{f}}$ is the shape parameter calculated as $1 / \mathrm{t}$ for $\mathrm{E}_{\mathrm{L} \text { and }} \mathrm{w} / \mathrm{t}$ for $\mathrm{E}_{\mathrm{T}}$, where 1 , $\mathrm{t}$, and $\mathrm{w}$ are length thickness and width of the filler.

$$
\begin{gathered}
\frac{E}{E_{m}}=\frac{1+\xi \eta v_{f}}{1-\eta v_{f}} \\
\eta=\frac{\frac{E}{E_{m}-1}}{\frac{E_{f}}{E_{m}+\xi_{f}}}
\end{gathered}
$$

Mori-Tanaka model This model has developed based on the principle of Eshelby's inclusion model for elastic stress field.

$$
\frac{E_{L}}{E_{m}}=\frac{A_{O}}{A_{O}+v_{f}\left(A_{1}+v_{0} A_{2}\right)}
$$

$\frac{E_{T}}{E_{m}}=\frac{2 A_{0}}{2 A_{O}+v_{f}\left(-2 A_{3}+\left(1-v_{o}\right) A_{4}+\left(1+v_{o}\right) A_{5} A_{0}\right)}$

Where $E_{m}$ is young's modulus of the matrix

$A_{O} \ldots A_{5}$ are the tensors

$\mathrm{v}$ is volume fraction.

\section{Thermodynamics of nanocomposite simulation}

It surely controls the reaction of polymer- nanocomposites and up to which extends the reaction proceeds. The interdicted various structural aspects affect the bonding potential among the nanoparticle- nanoparticle and polymernanoparticle. To hold control in the nanocomposites Synthesis and performance improvement, one needs to have a degree of control and provide favourable conditions to the precursors of nanocomposites during its preparation. [11-15]. Generally, Stability term is directly associated with the thermodynamics evaluation of the whole scenario of nanocomposites. It affects due to the dispersion effect of nanoparticles covering a range of entropy, enthalpy and freeenergy changes at equilibrium, which are thoroughly explained by the Mean-field model 16] 


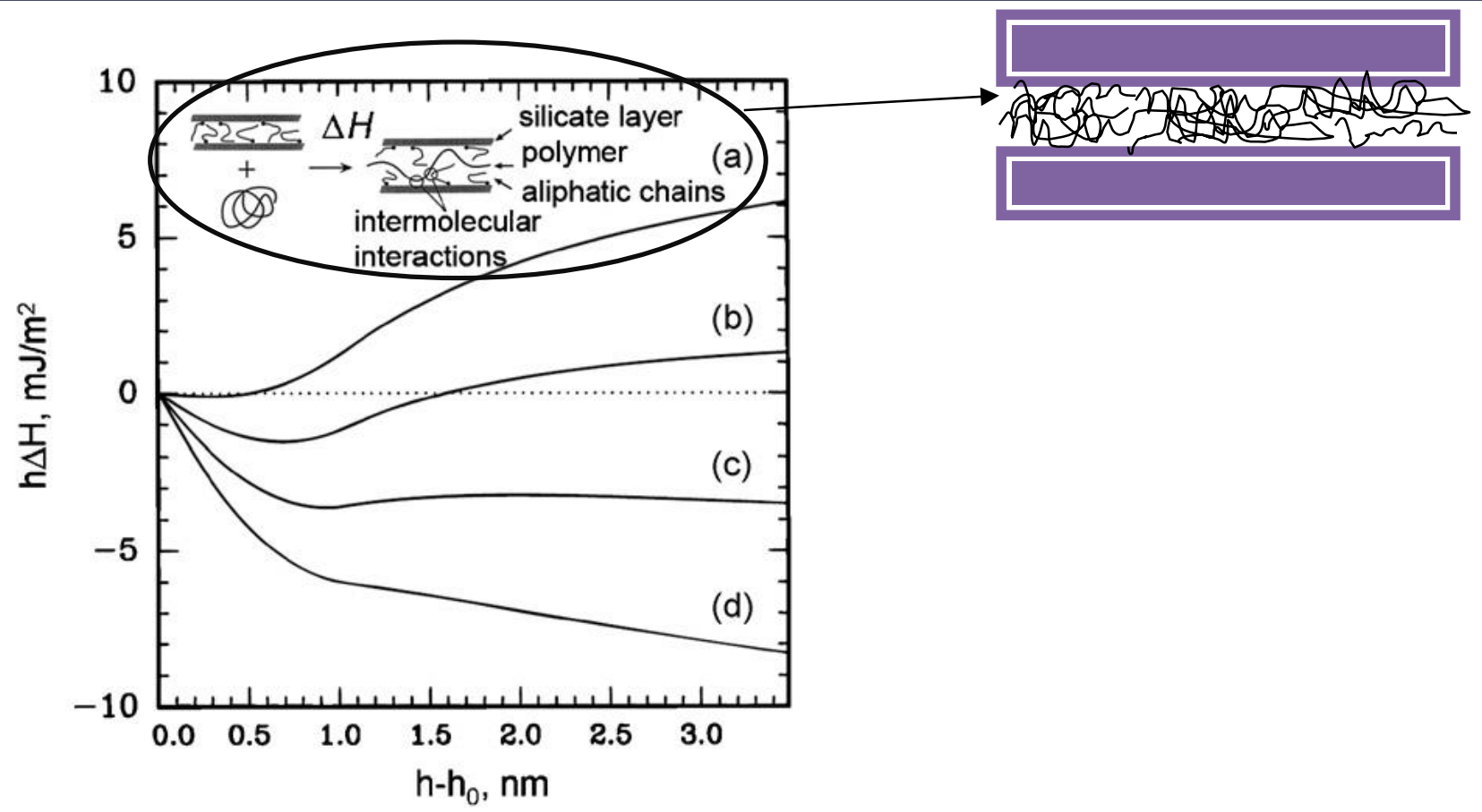

Fig. 2 Free-energy change (DH) of polymer-organoclay mixtures as a function of clay gallery separation under different intercalation strength (e) between polymer-organoclay: (a) e 1/4 0; (b) e 1/4_ 4 ; (c) e $1 / 4$

The inset shows (Fig. 2) the intercalation process of polymer in organoclay (Representing the free-energy profile describing intercalation and exfoliation of polymer into silicate layers.)

Studies of thermodynamics in nanocomposites have been studied at experimental and theoretical levels. For simulation technique initially, the free-energy (fig 2) change with the dispersion of platelets in the matrix of polymer is handled with a few thermodynamically terms such as

$\Delta \mathrm{U}$ - thermodynamically it represents the Internal energy explained by first law relations; for simulation, it represents the molecular interaction.

$\Delta \mathrm{S}$ - Well known and important parameter symbol entropy explained by the third law of thermodynamics; in the simulation, it interprets the configurationally changes for different components. For the interpretation of the internal changes, basic symbols interplay their roles.

Now, introducing the ruling equation on examining the thermodynamic reliability of the simulation system

$$
\text { subj= } \Delta U-T \Delta S=H_{j}-H_{i}
$$

Where $H_{i}$-- represents the initial clay platelets

$$
\begin{aligned}
& H_{j} \text {-Rrefers to the final clay platelets } \\
& \mathrm{T}-\text { Temperature }
\end{aligned}
$$

Here, conditions are if $\Delta \mathrm{H}$ comes out to be less than 0 then the intercalation process is favorable otherwise his loose state is adapted. Further, the interpretation has been done based on calculations, in which 3 isolated states of equilibrium have been developed. The gain in entropy tends to cover suppresses in the polymer confinement in clay gallery spacing. Kim et al [17] performed the Molecular Dynamics on clay-nylon6,6 and clay-nylon6 nanocomposites. They proposed that the intercalation among the polymer and modified organoclay platelets can be achieved with the help of exothermic heat of mixing; they have examined the simulation on the governing principle of statically thermodynamically equilibrium. 
The MD simulations are effective over the comparable range of radius of nanoparticle and gyration radius and segmental length. Smith et al [18] studied the interactions between the nanoparticle-nanoparticle when immersed in the polymer matrix with the help of Coarse-grained MD simulation. To read the interpretation Radial distribution function was manipulated with which few peaks have been obtained as a resulted fig.3.The graph explains the development of weak interaction between polymer and nanoparticle which may lead to the aggregation of nanoparticles in the polymer matrix indicated by the sharp peak, while the strong attraction between nanoparticle-polymer interactions can enhance the settlement of polymer over the clay that leads to the dispersion of clay, Infect Ozmul and PICU as well stimulate the distances for calculation purposes.

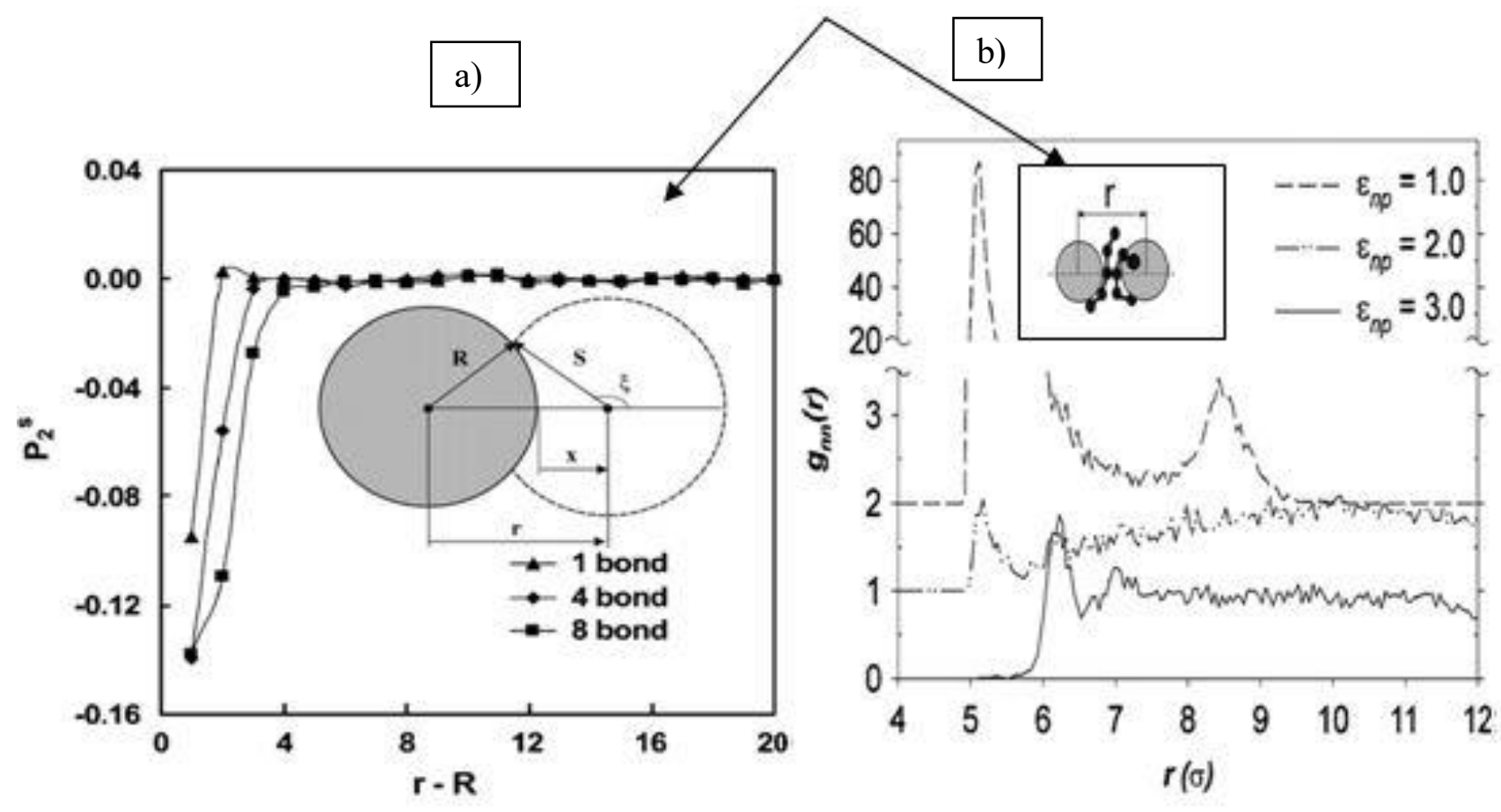

Fig. 3. a) Average segment orientation $P 2$ s as a function of the distance (r-R) from the surface of the particle. b) The nanoparticle-nanoparticle radial distribution functions under different interaction parameter values (enp), data are offset vertically by 2.0 and 1.0 for enp $1 / 41.0$ and enp $1 / 42.0$, respectively.

Figure 3 shows the schematics of allowable directions for the segment end-to-end vector in the vicinity of a curved wall. Here $\mathrm{R}$ is the radius of curvature and $\mathrm{S}$ represents the bulk length of the end-to-end vector considered. b) The nanoparticle-nanoparticle radial distribution functions under different interaction parameter values (enp), data are offset vertically by 2.0 and 1.0 for enp $1 / 41.0$ and enp $1 / 42.0$, respectively. He has proposed step by step formulation in simulation and conclusion clear the picture Balazs et al [19] they have come up with the solutions to the limitations of the mean-field model, used to explain the role of thermodynamics in nanocomposites simulations. Balazs [20,21] and his co-workers developed new series of model extracted from Fleer [22,23] and Cardigan[24] the polymer and surfactant coupling conformation affects individual configurations, have explained through SCF calculation which results in a similar conclusion as given by the Mean-field model for clay-polymer interaction model[17] the increased interaction between polymer-nanoparticles the free-energy shifts from positive to negative which conform the intercalation and exfoliation(fig.2). 

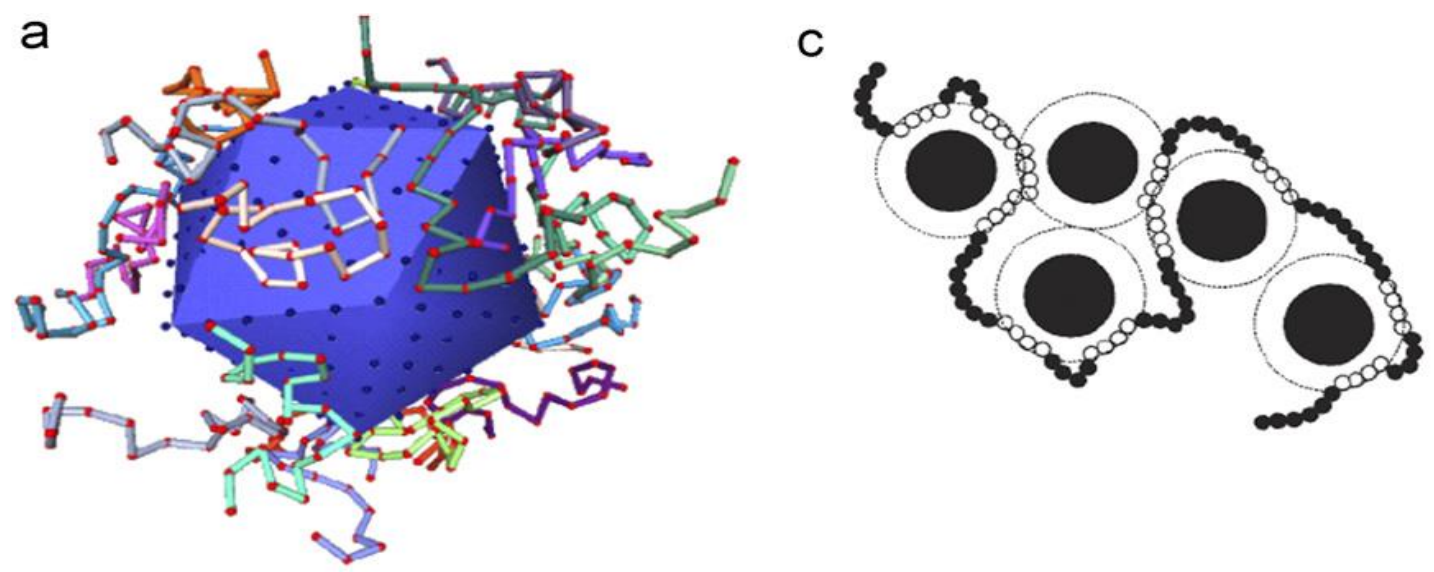

b

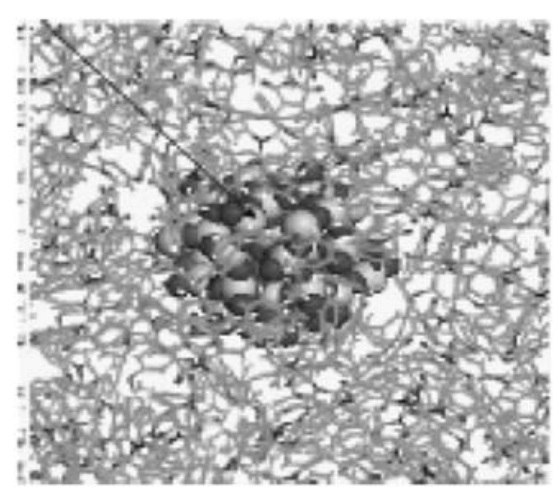

d

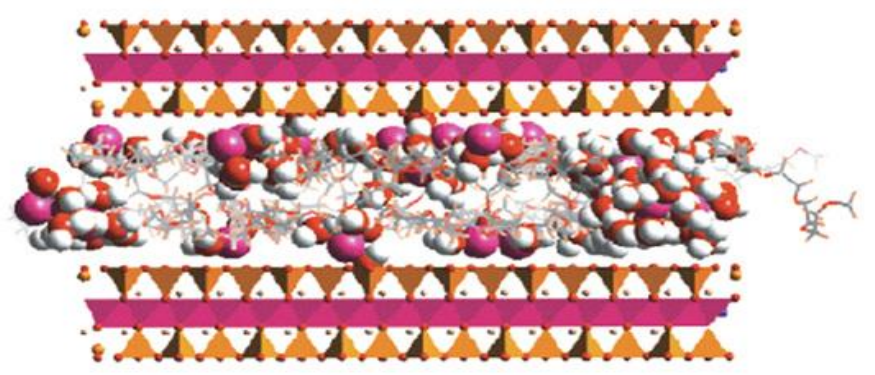

Fig. 4. Representative models of nanoparticle-reinforced polymer systems: (a) one spherical nanoparticle in polymer (b) one silica nanoparticle in polyamide (c) multiple nanoparticles in polymer and (d) polymer intercalated nanocomposite.

Further they investigate the effect of functionalized and non-functionalized polymers' chain lengths, the attachment of reactive groups at the end of the chain increases the degree of intercalation of the polymer-nanofiller which conforms to the thermodynamic stability of the system. Moreover, the ratio of their lengths also generates the phase boundaries among immiscible and level of dispersion of precursors in nanocomposites. By taking the lead from the above conclusion (regarding the effect of aspect ratios). Groenewald et al; [25] studied the simulation on grafted clay particle and copolymer in which he generates the lamellar structure, in which they concluded that the aspect ratio is directly proportional to the interaction/intercalation/degree of mixing in nanocomposites.

Tanka et al. [26] and Fermeglia et al. [27] put forward the same conclusions, from their respective simulations which surely strengthen the reliability of the simulation hierarchy and conditions. The first conclusion explains the role of surfactant volume, the increase in its volume surely raises the binding energy between polymer-surfactant and claysurfactant individually but the binding energy between the polymer-nanoparticle has decreased. Secondly, the attached polar groups with surfactants develop strong interaction energy with the polymer chain.

Goerke and co-workers [28] using MD simulation investigated the binding interaction factors on Polylactic Acid -Hydroxyapatite and Polyethylene-Hydroxyapatite.They found that the thickness of HAp causes higher potential binding interactions compared to thin HAp. A linear relationship developed among two major governing parameters i.e., binding energy and surface area. Coverage over the surface efficiently occurs on both the systems but the electrostatic guided interaction of PLA-HAp is stronger than PE-HAp. 


\section{Kinetics of nanocomposite}

Kinetics is the key to analyzing the formation dynamics and rate of polymer nanocomposites. This term is very much less understood and explained, the questions on how does polymer initially enter the nanofiller galleries. Similar to the above question many more steps are needed to be investigated, in which the behavior of the clay gallery opening to the attack of the polymer $[29,30]$ what kind of mechanisms do they follow? To which level the penetration is handled by the clay gallery/polymer matrix. More questions like these need to be answered. Loring et al [31,32] investigated the kinetics of polymer-nanofiller/clay nanocomposite on the coarse-grained Molecular dynamics simulation technique and through the flow of nanocomposite over the evacuated slit, which showed the restriction inflow due to the increase of polymer surface empathy.

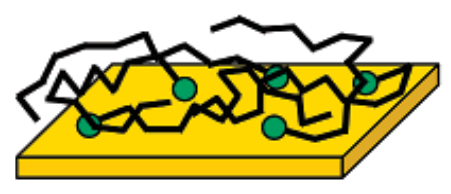

$0 \leq \lambda_{0} \leq 0.2$

Amorphous (25-30\% gauche)

No phase transitions (Reorientation of the alkyl chains upon first heating)

(a)

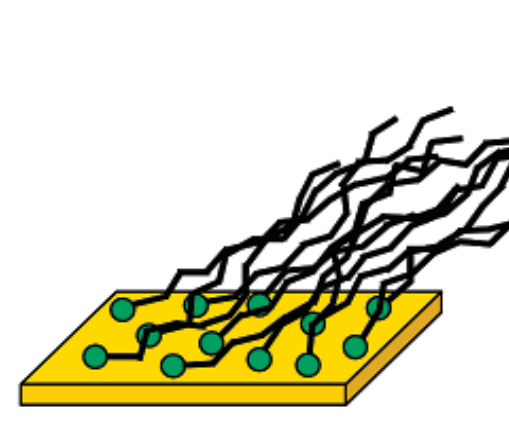

$0.2 \leq \lambda_{0} \leq 0.75$

Semi-crystalline (ca. $20 \%$ gauche)

Reversible phase transitions observed

(b)

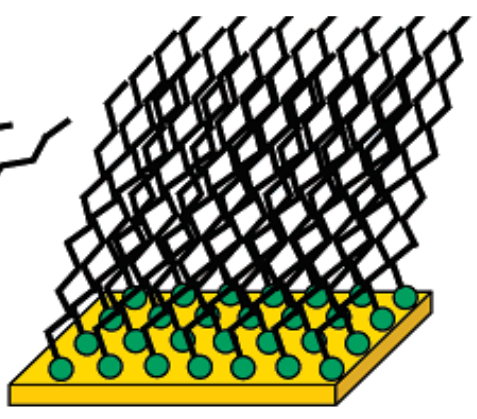

$0.75 \leq \lambda_{0} \leq 1$

Quasi-crystalline

(<10\% gauche)

\section{No phase transitions}

(c)

Fig. 5. Snapshot of Range of homogeneous alkyl layers (chain length g C10) on even surfaces includes (a) disordered chains oriented parallel to the surface, (b) intermediately ordered chains with an intermediate collective tilt angle, and (c) nearly vertically oriented, all-anti-configured chains. Significant reversible thermal transitions are found only in case $b$.

Experimental results have also given the same conclusion. They may find some relations among the kinetics of the polymer-clay nanocomposite but due to ignoring the main features such as a) the effect of the surfactant and b) swelling of slit [33]. The interpretation became less reliable to carry forward. They need more investigation, explained the intermediate polymer surface affinity is enough for intercalation and exfoliation. Also, the rate of flow reduces with the increase in the polymer molecular length [34]

Ginzburg et al;[35] have proposed a critical model which explains the change in the structure of the clay platelets and shear force for the intercalation process. They found that the high external shear force can result in the elongation and kink of clay platelets with the balancing in bending elongation. Further, Gendelman et al;[36] enhanced the explanation of the Ginzburg model by developing an improved model that investigates the kinetics of the process with the initial input of the clay gallery opening to the polymer induction. The initial stages rule the dispersion of the clay the applied external force for the intercalation process is enough for the propagation of bending with appropriate pace.

The effect of length parameter furnished by the [28] in fig.5 the study of PLA-HAp and PE-HAp comparative study of interaction mechanism showed that the longer polymer chains enhance the polymer-polymer interaction but the filler- 
polymer interactions weaken. For studying the kinetics of the polymer nanocomposites, the focus on the changes that develop in speed and morphology with phase separation has been taken into an account. Balazs et al; [37,38] developed a multiscale model by combining the various models such as coarse-grained molecular dynamics, the mesoscopic model for discrete particles. They came to know that the solid particles affect the orientation and flow of the different phases. Their mean-field model helps them to understand the importance of the diffusion coefficient and the particle concentration. Under shear force, the system initially perfume like an isotropic manner then grows swiftly in the direction of shear and later it got slow down in a perpendicular direction to the applied shear.
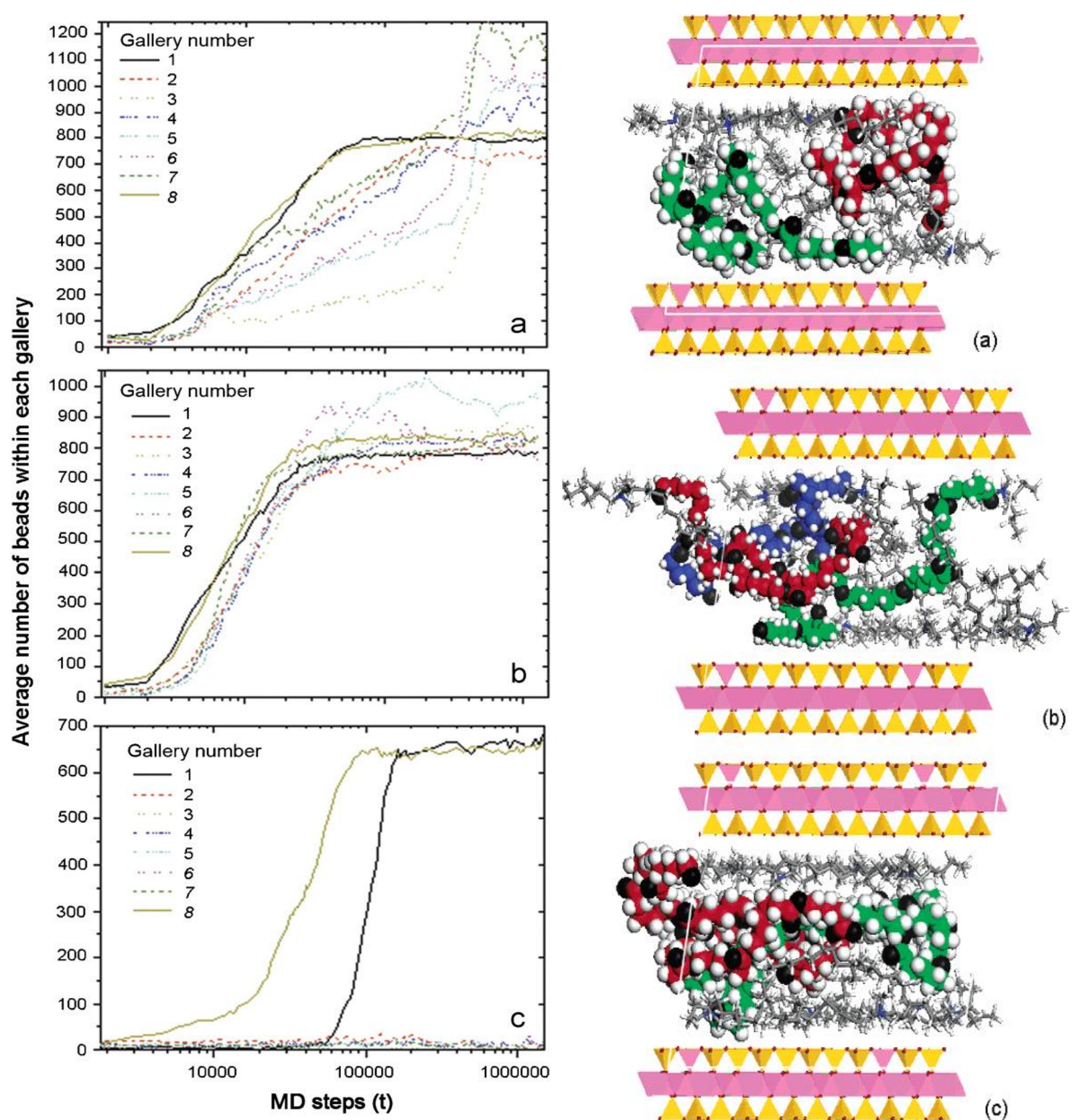

Fig. 6. Snapshot of an average number of beads in each gallery of (a) A25-clay, (b) A75-clay, and (c) A01-clay and the simulations of (a) PNC system I, (b) PNC system II, and (c) PNC system III obtained after 250 ps of MD calculations(on the right side). 
Galleries (Fig. 6) represent the spacing between the top two and bottom two sheets, respectively [18,78]. Reproduced Vaia and his co-workers (on the left side). the simulations of (a) PNC system I, (b) PNC system II, and (c) PNC system III obtained after $250 \mathrm{ps}$ of MD calculations. The clay layers are represented by one octahedral sheet sandwiched between two tetrahedral sheets. In the gallery, the surfactants are represented with sticks and the PCL chains with spheres; the carbon atoms are in green, red, and blue, the oxygen atoms in black, and the hydrogen atoms in white. Lazzaroni and co-workers (on the right side).

Anderson et al;[39] worked on the coarse-grained molecular dynamics simulation to study the different components of fluids and organoclays. They examine the interaction pattern among polymer and clays(fig.4). They introduced two types of fluid based on intramolecular attractive forces in type A and type B beads respectively. Which were tried to co-relate with Lazzaroni's work on PNCS' simulation shown in above fig.6. Common term result comes up in all fluids in which two outermost layers of the flexible clay infuse with the fluid beads for the intercalation. A25 (25\% type A beads) showed dispersion of beads into three-layer. A01 (1\% type A) entertain only the outermost layer for the intercalation and A75 (75\% type A) act similarly as A25 similar pattern followed up by PNC systems.

\section{Nanocomposite molecule structure and dynamics properties}

Estimating the improvement in the ultimate material formed with the accurate addition of the precursors of nanocomposite has a major influence on its structure. Experimentally, the information on its structural orientation can only study from various testing techniques such as SAXS, WAXS, XRD, FTIR, NMR etcetera but it's difficult to develop the details of the gallery and its interfaces. The control over its structure and morphology can help in designing the desired material according to its application in the real world which cannot be able to get from experimental results [4042]. Maybe this requirement made the high demand for simulation over the inner dynamics of the clay and the polymer irrespective of their individual. As molecular chains behave differently in the spacing of the clay gallery in comparison to the bulk. Indeed, it has been simulated that the polymer impinges itself in the clay gallery vicinity to form a parallel lamellar structure whereas, its alignment and orientation depend upon the intermolecular interaction among the particles. The addition of nanoparticles develops the change in the structure of Polymer.

The properties have examined includes chain orientation, alignment, density profiles, nanofiller loading, interaction potential. In addition to nanofiller of any dimension in the polymer chain, its volume fraction in correspond to the polymer is always smaller than the bulk. The addition of nanoparticles in polymer chain dimensions equivalently becomes smaller in comparison to bulk system studied by Vacatello and market al[43] keeping the assumption that the effect of non-interacting chains in the system is almost negligible to study[44,45] MC and MD simulation used to examine the molecular structure and morphology of the nanofiller embedded polymer matrix the radius of the nanofiller has a critical value above or below which the embedment in the polymer chain of the nanofiller becomes perturbed. That critical radius is a root mean square gyration radius of the molecular chain said by Ozmusul et al. [47] Further, he has performed MC simulation to study the dependency of the polymer structure at the bond scale and chain scale affected by chain length, density, surface curvature and size. At the bond scale, the end chains agglomerate and become independent of surface curvature whereas at chain scale the chains usually rotate at their semi-axis reaching towards the center of mass of nanofiller especially ellipsoidal chains. Then he reached to investigate over the static structure of the 
polymer nanocomposite interaction using the MC simulation technique [48] he has used a few terms to explain the chains segments such as bridges, daggling, loops and trains segments. The transient network is being formed in polymer nanocomposite structure, the increase in the addition of nanofiller because the increase in bridging that may be due to the mean distance between fillers. Again, the Vacatello perform his investigation on the structure of polymer nanocomposite through the approach of segment suspend by Vaia; (fig.5-6) he has considered the segments named as Interface segment, loop segment and bridge segment. He has generated the data that guide the experimental system as the dependency of the changes in structure largely depends upon the symphony of the system. Vacetallo [49] has studied a few more possibilities and ways to find out the actual theory behind its structure gradient. He proposed a few chains dependent situations that affect the structure and surface morphology changes likewise the formation of free chains due to the difference in the chain lengths of the polymer and nanofiller to overcome this problem he has again simulate at chain scale where he concluded that the interaction energy creates the phantom chain characteristics. They have sampled the randomly distributed large and small nanoparticles in the polymer chains. Later the change in the composition, size, interface etc affect the segments of the polymer nanocomposite interfaces and integration becomes quite predictable. Breu et al;[52] first time use the lattice energy minimization technique to study the 2-D structure (clay platelets) in polymer nanocomposite for which study at the atomic level is desired. His study has concluded that the intercalation structure got affected by the complex interchange of clay-polymer and polymer-polymer interactions. Then comes Manians [53-56] with his co-workers for the investigation at the atomic level using MC and MD simulation technique where they use PEO-MMT interaction structure. They found that the Polymer arranged itself as liquid (layered) with the clay nanoparticle platelets.

To study the structure of nanocomposite at exfoliation and intercalation stages, Lazzarini et al; [56,57] targeted the PCL polymer entrenched with clay platelets. For exfoliation plots of density profiles helped in concluding the five-layer structure fig. 7 Where they obtain the peaks of different higher densities in comparison to Amorphous and crystalline PCL. The highest peak in density profile refers to the exfoliated interface between polymers - clay, whereas the lowered density peaks refer to the presence of the shielding effect in the bulk system. The PCL polymer may arrange a bridging structure which causes the restriction in exfoliation, but the insitu process inhibits the effect. Katti $[59,60]$ has tested the results obtained from the experimental and theoretical study by MD simulation using density profiles same as Lazzaroni approach with his co-workers on nylon-6 and clay nanofiller system. Zeng et al; has also reported the layered structure of alkyl-ammonium a different increasing stage of arrangements forcing pack molecular structure with cation exchange capacity shown in fig.8. [61,62,63] Polyurethane nanocomposite has both hard and soft segments in its gallery [64] for its study phase separation is a very vital aspect to investigate at bulk separation which seems to be absent in polyurethane nanocomposite. 


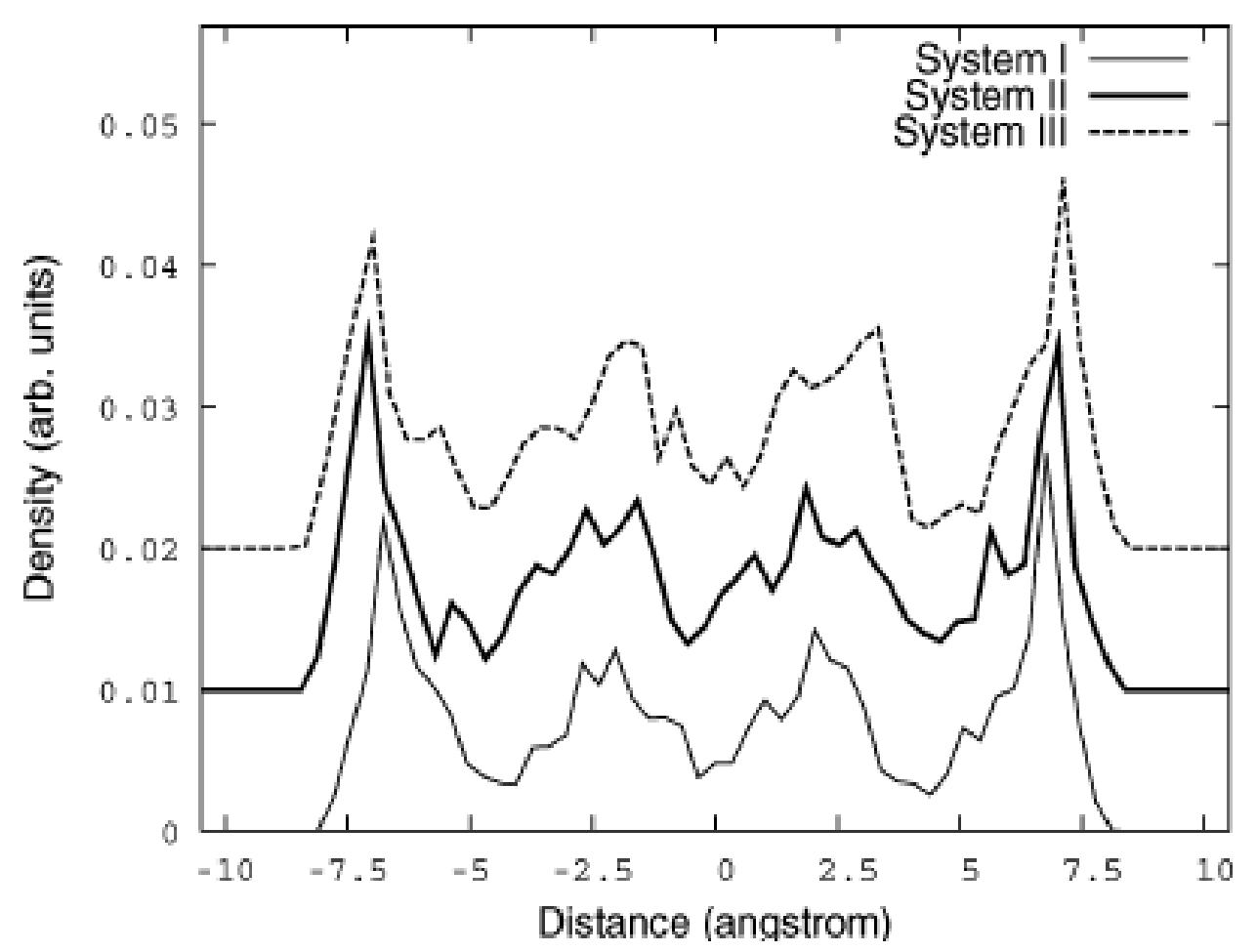

Fig. 7. Density profiles for the backbone atoms of the surfactants and PCL chains in the direction normal to the clay layers, reported for the PNC systems I, II, and III.

The positions of the two planes of basal oxygen atoms are at (10.5 $\AA$ ). For clarity, the profiles for systems II and III have been shifted by 0.01 and 0.02 along the $y$-axis, respectively. 

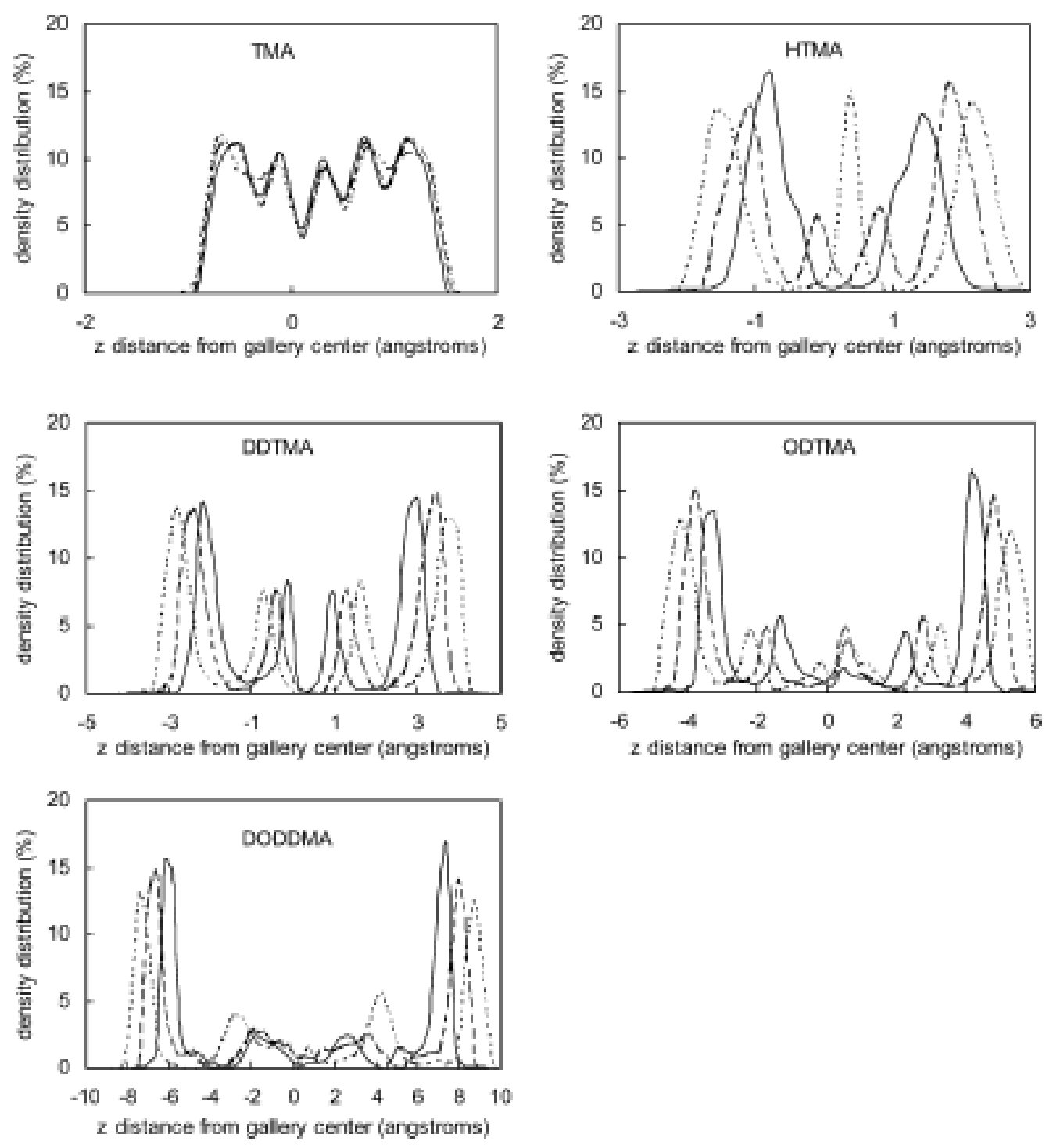

Fig. 8. Snapshot of methyl group density distribution of organoclays with different CEC and alkyl chain lengths as a function of z distance from gallery centre: solid curve, CEC 85 meq/100 g; dashed curve, CEC $102 \mathrm{meq} / 100 \mathrm{~g}$; dotted curve, CEC 119 $\mathrm{meq} / \mathbf{1 0 0} \mathrm{g}$.

Then Greenwell et al; [65] proposed the contribution of the amine group for the interaction in clay with the polymer in the intercalation also agrees with the results of paul et al. [63] they proposed that the ammonium groups are core activities in the interaction then amine functional group. Heinz et al ${ }^{[66]}$ worked in the alkyl-ammonium modified MMT using MD simulation and explored that the cation exchange capacity. A new approach of hybridizing the techniques has been done by sides et al. [67] by adding the SCF with DFT to overcome one other's drawbacks of calculation. Investigating the system through this simulation so it couldn't be restricted to the mean-field estimations implies to the SCF model, here the individual article is described through the discrete particle-based simulation. This combination makes a way to trace the individual particle in the system and examine their structure and morphology at equilibrium. It is solely believed that such a hybrid combination method surely plays an important role in determining the morphology of polymer-nanofiller interactions. 


\section{Nanocomposite rheological and processing behaviour}

The impact of the microparticle on the viscoelastic properties can be quantified in terms of volume fraction of particles but the study of nanoparticles is quite not developed. The rheological properties help in the simulation of the system to build control in its processibility to get desired ultimate product [68-70]. Nanoconfinement understanding is necessary which can be done only through the simulation techniques and modelling to manifest the optimizing conditions for processing. Studies have said that the nanoconfined homopolymer sows that the dependency of the shear stress over shear strain is linear up to some point then it turns out to be at the rating power of which refers to the strength of the surface interaction in the homopolymer [71-72]. The limitation of homogeneity has been removed by Manias et al. [56] by programming on the equilibrium MD simulation technique for investigating the rheological and dynamic behaviour of the nanoparticle entrenched films. Book et al [73] along with their co-workers have performed the simulation on the different shapes of the nanofillers for likewise solid rods. Disks, through DPD simulation technique et al;[74] investigated the rheological properties through examining the behavior of the end-functionalized chains in the matrix. It has been noticed that if the number of functionalized groups increase the response over the shear has also changed significantly $[75,76]$. Unfolding the dependency factors for the optimization of the processing technique which is reliable for the clayloading nanocomposites.

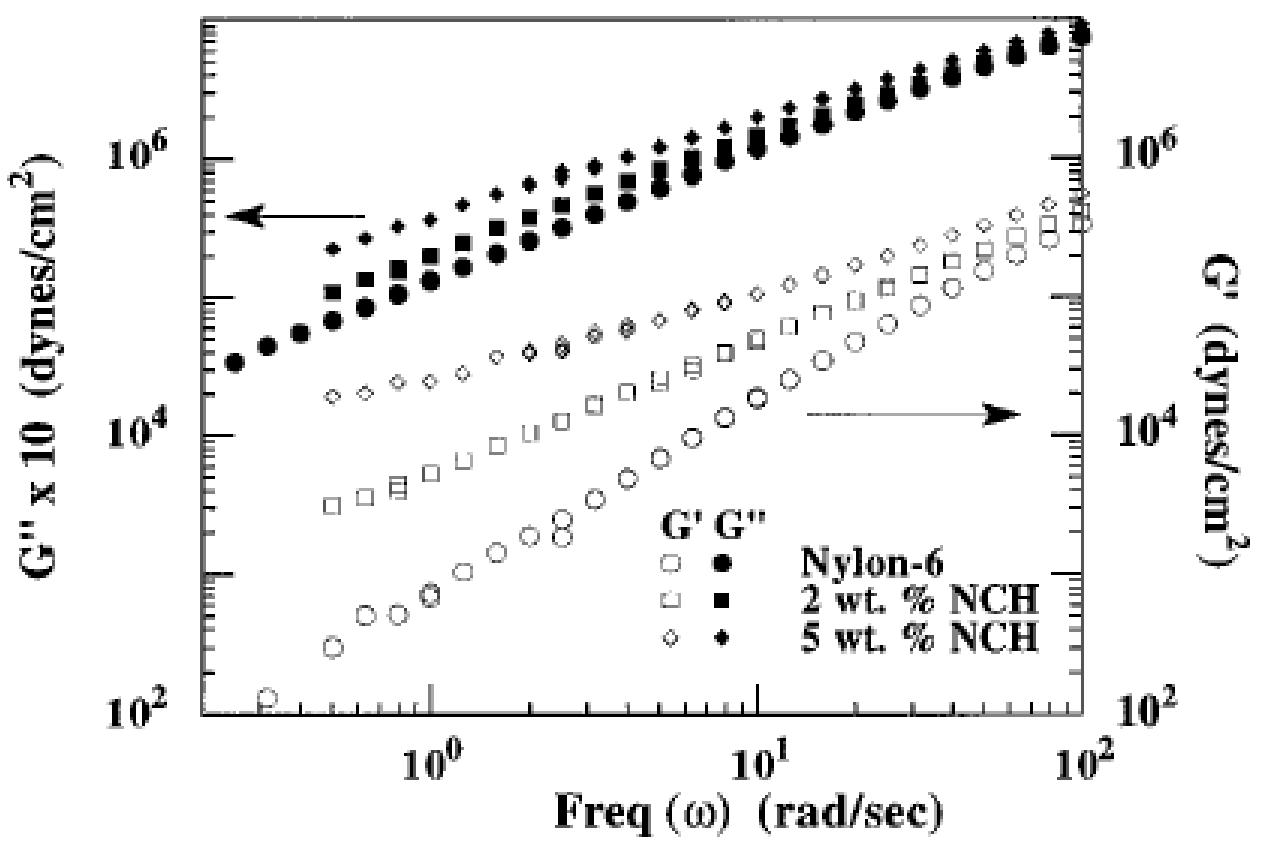

Fig. 9. Snapshot of small strain-amplitude oscillatory shear behaviour of nylon-6s montmorillonite end-tethered nanocomposites $(\mathrm{NCH})$ at $235^{\circ} \mathrm{C}$. Storage moduli $\left(G^{\prime}\right)$ and loss moduli $\left(G^{\prime}\right)$ as a function of oscillatory frequency $(\ddot{\partial})$ for pure nylon-6, 2 and 5 wt \% nanocomposite is shown.

Groups [77] constantly studied the intercalated clay nanocomposite (fig.9) to examine the effect of the percentage loading of the clay on the flow properties and it is found that the viscoelastic properties are independent of the anisotropic clay loading. Smith and co-workers [78] studied the viscoelastic nature of the nanoparticle nanocomposite using the MD 
simulation technique. Calculations for the shear stress relaxation modulus $\mathrm{G}(t)$ using time autocorrelation functions based on Einstein relations for shear tensor and viscosity. The relations for the dependency of the changing shear stress on the viscosity in nanocomposites have been studied through different variables such as interaction, interfacial areas and volume fraction. In contrast to other conventional reports, it showed dependency on the interaction. The values for shear modulus and viscosity rose for the nanoparticle-polymer nanocomposite.

Starr et al;[79] have studied the mechanism of the clustering of the nanoparticle and factors positively affecting the process. The shear viscosity for the dispersed nanofiller is higher than the cluster nanoparticle. The colloidal scattering enhances the rate of clustering in the polymer matrix as it resists the entering of the nanoparticle into the vicinity of the polymer matrix. Pramitysn and his co-worker [80] utilized the Coarse-Gained simulation technique has found the relations for the same extent of the shear stress and rheology for the composite and the composite suspension normal fluid. At different dilution states, the shear rheology is dominated by the shear-thinning fluid. The contribution of the Nanoparticle has more dominating over the shear rheology in comparison to the polymer.

Sarvestani and Picu [81] used a theoretical network approach to investigate the behavioural changes in the nanocomposite with the change in the low filler volume fraction and interaction between them. It was found that it strongly depends upon the lifespan of the nanoparticle-polymer junctions. Which showed the rubber-like flow at low shear then constant flow which turned to be shear thinning flow at high shear? The effect of the intercalated CNTs over the rheology of the nanocomposite has been reported by Song et al [82].

Wang along with his co-workers [83] worked on the Shear rheological properties of the CNFs in polystyrene matrix also the morphological changes occur through the melt-blended and the solvent-cast process which snapshots the Nanofibers positions on the shear rate through experiments. The model predicted the constant values for the viscosities on the application of the shear flows. Fibre length and polymer matrix play their role in normal stress differences.

\section{Nanocomposite mechanical properties}

Coming to the mechanical behaviour of the nanocomposite on the addition of filler to the pristine polymer. A very small amount of the nanofiller can put a significant impact on its mechanical properties. It enhances its strength and toughness. It can resist the line of failure and make it harder to crack [84-85]. Additional to the property's improvement is mainly done by the layer arrangements formed by the toting up of nanofiller in the pristine polymer.

Generally, it is accepted that the particle size, aspect ratio, shape of the system, interaction among the polymer-nanofiller and in between them. Also, the intercalation of the nanofiller affects the behaviour of the ultimate nanocomposite. In the collection of plenty of nanofiller, clay platelets possess [86-90] more potential to reinforce the polymer in comparison to other fillers for mechanical testing. Reinforcement of the polymer over the very minimal amount of nanofiller loading for enhancing its strength and force bearing characterization makes it's more suitable for engineering material for wide applications [87].

A wide range of length and time scales have been used for the simulation of the ultimate material. Mechanical properties can be calculated by simulation techniques accordingly to the size scale like for molecular scale (MD, MC etc), HalpinTsai, FEM, etc for macro scale to micro and multiscale (combining them). Continuum models also help in the ripe out the analysis over the micromechanism.MD simulation is been used in abundance for the analysis of the nanocomposite structure and morphology that enhances its properties. The shear stress-bearing of polymer enhanced by the addition of 
nanotubes, its high magnitude of the interface formed of light chemical bonds makes its efficient cross-linking arrangement.
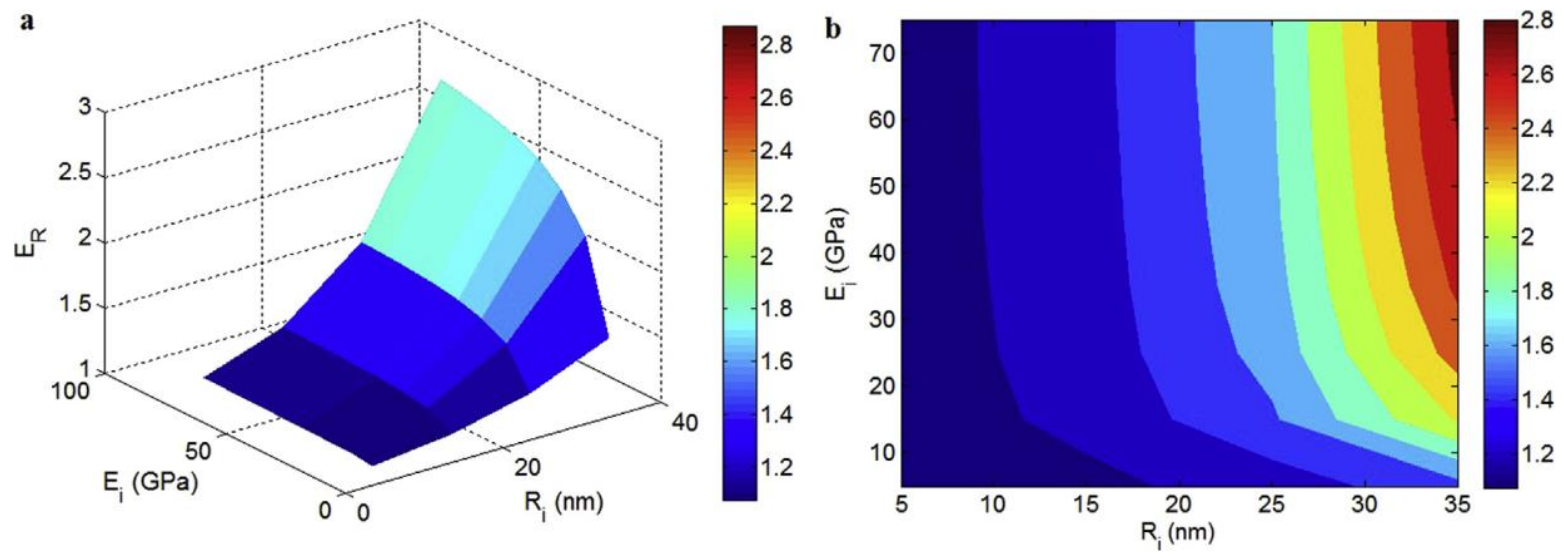

Fig. 10. Snapshot of (a) The influences of " $R_{i}$ " and " $E_{i}$ " on " $E_{R}$ " by the developed model at $4 f 1 / 40.02, R 1 / 420 \mathrm{~nm}, E_{\mathrm{m}} 1 / 42 \mathrm{GPa}$ and $E_{f} 1 / 460 \mathrm{GPa}$. (b) $E_{R}$ " as a function of " $R$ " and " $E_{f}$ " according to the developed model at $4 f 1 / 40.02, E_{m} 1 / 42$ GPa, $R_{i} 1 / 420$ $n m$ and $E_{i} 1 / 440$ GPa.

The surface areas of the filler effect linearly the work to failure $\left(\mathrm{W}^{*}\right)$ along with the interaction in between polymernanofiller in comparison to the pristine polymer. The same size of the nanofiller as polymer has been examined to study the behavior at work at failure [91]

Stress transferring is also investigated through molecular modelling simulation technique at the interface of polymer matrix adding to its combined efforts have made over the interfacial pattern of CNT-polymer on shear stress which was found to be obtaining higher magnitude than fibre-polymer. Load transferring of SWCNT parallel bundle has been studied by Qian and his co-workers [92] Twisting of the closely packed bundle showed the improvement of the loadbearing capacity. Nanotubes interaction with epoxy resins enhances the load transferring property reported by Gou and his co-workers [93,94]

Polyethene has very high tensile strength and when it is reinforced with the addition of CNTs, its behavior upon mechanical properties has been studied by Monakshi and her co-workers [95] utilizing the MM simulation technique. Long CNT tubes couldn't able to raise higher mechanical properties than pristine polyethylene properties. Whereas, the short-range CNTs have significantly improved their tensile, strength, and stiffness. Lohri and Yao [96] focused on the interphase adhesion of CNTs by using MM simulation. They examine the binding energy of the polymer and nanotubes. During the calculation, a few new approaches came up explained in mathematical terms. They found the structure and bonds play a vital role in providing stiffness strength at the interface. The helical structure is an essential arrangement. Later Zheng, Prathab [97] did an investigation of the CNTs-polymer interfaces by wrapping the polymer over the nanofiller which someway showed good interaction among them. Also, the behavior of metal oxides as nanofiller has been examined by MD computer simulation. The investigation on ethylene-vinyl alcohol copolymer organoclay has 
revealed that the hydrogen bonding formation between the amine and hydroxyl oxygen this bond twisted to be a stronger bond.

The improvement in mechanical properties can be observed upon low loading of nanofiller in polymer-particle nanocomposite [98]. The strong bond in the nanocomposite matrix made it solid-like possesses a bond that has approximately infinite relaxation time. Song et al. [99] gave the factor i.e., relaxation time between the bond of the polymer-nanofiller interaction helps in delaying the fracture on a time scale which further investigated by the Kalfus et al. on the poly (vinyl acetate)-Hydroxyapatite nanocomposite that reported that the stress storage module recovery time was controlled by the adsorbed polymer on the particle surface.

\section{Continuum models}

The orientation, geometry, directional, volume fraction in the polymer matrix has been studied through different continuum models. Paul et al. studied the effect of the factors of nanofiller over nylon-6 induced with organoclay and glass fibres by utilizing Chow [100], Halpin-Tsai model and Mori-Tanka model arguable conclusion came up from the calculations as they nylon- 6 is subjected to the intercalated high moduli carried along high aspect ratio of layered clay to reinforce in different directions. But they observed a drastic decrease in the functionalization efficiency of the nanocomposite due to an increase in the gallery spacing and clay platelets loading better heat distortion achieved of the nylon-6-clay over the high aspect ratio and high young's modulus. Luo et al. developed the 3-D phase model for examining the exfoliation and intercalation states of the mechanism polymer-nanofiller nanocomposite. Mori-Tanka simulation has been used for the calculation of the modulus based on various factors a) high filler dispersion b) stiffness improvement on the exfoliation rational c) aspect ratio of the nanofiller d) a prefrontal intercalation gallery spacing unless it forms the cluster. Zeng and co-workers [101] worked on the Mori-Tanka model for the study of the elastic property of the static and dynamic of the nanorod-polymer nanocomposite.

By utilizing the Halpin-Tsai model,[102] provide an analytical solution for the elastic equation. They observed a few important aspects in the micro mechanism like buckling of clay platelets, clay exfoliation and improper-sized platelets. They have critical alignment to achieve high strength and reinforcement efficiency. Whereas constant failure theory reported by the Guz et al; [103] developed a model on the micro buckling definition and followed two major approaches a) work on homogeneous model b) equivalent portion of the anisotropic model. Road and co-workers [104] studied the dynamics behavior of the aggregation of the particle at low, semi and full levels using the DPD model. This showed the direct effect of the filler-filler interaction $n$ the shear modulus. Further Buxton with Balazs [105] utilized the latticespring model for studying the filler structural and geometric aspects. Polymer reinforcement with the platelets increases its functionalization efficiency in comparison to the sphere nanofiller form inhomogeneity in the polymer nanocomposite matrix.

Many hybrid models have been designed to study the multilevel properties and factors of controlling by Li et al. [106]. Tensile creep has been studied on titanium dioxide/clay polyamide nanocomposite through empirical power law and Burger creeps' mode satisfying relation reported, the increase in creep resistance at the addition of nanofiller. Habib et al. [107] worked on the comparison of the mechanical behavior at two different platforms micromechanical Mori-Tanka and FEM predicate that the clay loading up to 5 weight percent volume fraction raises the level of stiffness and after that, the stiffness aspect has not much improvement. 
Zare et al; [108] have worked on interface properties prediction using the Pulkanzy model of CPN by yield strength concept. The dependency of the 'B' interface parameter has been observed in his study. The model obtained a minimum value of ' $\mathrm{B}$ ' interface parameter i.e 2.4 below which the strength, stress at interface doesn't show any significant relation at both experimental and computer simulation levels(fig.10). Then he investigates developing the Halpin-Tsai model for polymer nanocomposite, he focused on interphase properties and spherical nanofiller particles at both experimental and simulation platforms. It was found that the volume fraction of interphase is higher than the value of the volume fraction of filler at different filler loading which means the volume fraction has occupied a significant area in the polymer nanocomposite matrix $[109,110]$. Also, the thickest interphase holds a good agreement with experimental results. The role of the radius of interface holds direct relation with young's modulus of modulus.

\section{Multiscale strategy for modelling nanocomposite}

\section{Challenges}

The main focus over the computational techniques rises because of the reliable and accurate prediction of new material and their behaviour. Studying the critical parameters experimentally becomes difficult to determine whereas, computer simulation provides a better model with rapid and exact possibilities. That helps in designing the experimental conditions and parameters to obtain the almost exact outcomes. The multiscale modeling bridges the gaps of simulation between the different scales of models from molecular to mesoscale levels working over the broad range of length and timescale. With multiscale modeling puts forward a few challenges with its processing that should be seamlessly and smooth to perform. Satisfying the first level model allows the system to set its outputs to other level inputs for multiscaling programming. MD simulation has become an almost classical technique because of its atomistic level predication in the arrangement of multiscale modeling but along with-it QM becomes very large and difficult. New strategies need to introduce efficiently for the ultimate material properties at various scales and relations among them, loading at nanoscale improve the material performance at the macro scale.

\section{Approaches}

Multiscale modeling is followed up through three kinds of different paths as Sequential, Adaptive resolution and concurrent approaches. Sequential approach, a bridging technique between the calculations carried from one stage to another stage [111,112], this way it follows up over small scales and bridging the larger picture. This approach proved to be very effective and beneficial for knowing the material behaviour at various levels. Ramirez-Hernandez along with his co-workers [113] have practiced this approach at the atomic level. Covering a large range of length and time scales this path is a very appreciable technique. On another hand, the Adaptive resolution approach has recently added an approach the molecule of the subject can shift their position freely in the domain. Liu has investigated this approach and could able to reach out to the different assemblies of the subject through this approach and studied the Brownian motion. The co-current approach is also termed as handshaking path and it is linked aiming at different scale models. Covering the rigid techniques smoothly and linking them at different scales helps in normalizing the statistical behaviour. This technique needs to be investigated much deeper for experiencing its benefits. Ortiz [117] have done work on this approach based on atomistic continuum handshaking. Where the atomic details are filled at nanostructure and others are put under continuum within finite element framework. These approaches can be performed through different routes to prepare their pseudo-DNA systems such as polymer networks, semiflexible polymers, polymer Entanglements, Tubes 
and Knots, nanocomposites and topology. Megarioties et al,[114] worked on polymer networks through slip springs as a backbone for the enlarged polymer scale by MC Simulation to improve its thermal properties. Multiscale modeling is actively performed because it merges one other methods' limitations and enhances the efficiency of simulation performance at the cumulative site. Understanding on static behavior of polymer nanocomposite is still needed more focus. Different assembly patterns and respective behaviour change of polymer nanocomposites on the addition of a small amount of nanofiller to it. Currently, many kinds of research have been performed hybrid / multiscale modelling to develop the investigation on structure from every corner. The heretical investigation from each level and now posted may multiscale modelling investigation also in which he has combined DFT with SCF1[116], also worked in co-polymer by addition the effects of CHC-BD-LSM simulation techniques ad may more to read. Borodin et al; [115] have proposed MD simulation for stress-relaxation and material point simulation methodology in which he has worked on viscoelastic behaviour, validate them from his simulation calculations and performed polymer-bead mean square displacement. Combining models with the help of bridging techniques surely helps in the release of the overall picture of the material from physics, mechanical and many other properties.

Declaration of Competing Interest: The authors declare that they have no known competing financial interests or personal relationships that could have appeared to influence the work reported in this paper.

\section{References}

1. Segad, M.; Jönsson, B.; Åkesson, T.; Cabane, B. Ca/Na Montmorillonite: Structure, Forces and Swelling Properties. Langmuir 2010, 26, 5782-5790.

2. Zhu, T.T.; Zhou, C.H.; Kabwe, F.B.; Wu, Q.Q.; Li, C.S.; Zhang, J.R. Exfoliation of montmorillonite and related properties of clay/polymer nanocomposites. Appl. Clay Sci. 2019, 169, 48-66.

3. Jayrajsinh, S.; Shankar, G.; Agrawal, Y.K.; Bakre, L. Montmorillonite nanoclay as a multifaceted drug-delivery carrier: A review. J.Drug Deliv. Sci. Technol. 2017, 39, 200-209.

4. El Ouardi, M.; Laabd, M.; Oualid, H.A.; Brahmi, Y.; Abaamrane, A.; Elouahli, A.; Addi, A.A.; Laknifli, A. Efficient removal of p-nitrophenol from water using montmorillonite clay: Insights into the adsorption mechanism, process optimization, and regeneration. Environ. Sci. Pollut. Res. 2019, 26, 19615-19631.

5. Vidotti, S.E.; Chinellato, A.C.; Boesel, L.F.; Pessan, L.A. Poly (ethylene terephthalate)-organoclay nanocomposites: Morphological, thermal and barrier properties. J. Metastable Nanocrystalline Mater. 2004, 22, 57-64.

6. L. Fogelström, E. Malmström, M. Johansson, A. HultHard and flexible nanocomposite coatings using nanoclay-filled hyperbranched polymers ACS Appl. Mater. Interfaces, 2 (2010), pp. 1679-1684.

7. G. Verma, A. Kaushik, A.K. Ghosh. Nano-interfaces between clay platelets and polyurethane hard segments in spray-coated automotive nanocomposites.Prog. Org. Coat., 99(2016), Pages 282-294

8. G. Verma, A. Kaushik, A.K. GhoshComparative assessment of nano-morphology and properties of spray-coated clear polyurethane coatings reinforced with different organoclays. Prog. Org. Coat., 76 (2013), pp. 1046-1056

9. G. Verma, A. Kaushik, A.K. GhoshPreparation, characterization and properties of organoclay reinforced polyurethane nanocomposite coatings.J. Plast. Film Sheeting, 29 (2012), pp. 56-77.

10. Kinloch IA, Suhr J, Lou J, Young RJ, Ajayan PM. Composites with carbon nanotubes and graphene:An outlook. Science 2018;362(6414):547-53.

11. Li X, Sun M, Shan C, Chen Q, Wei X. Mechanical properties of 2D materials studied by in situ microscopy techniques. Adv Mater Interfaces 2018;5(5).

12. Liechti KM. Characterizing the Interfacial Behavior of 2D Materials: a Review. Exp Mech 
2019;59(3): 395-412.

13. Hu KS, Kulkarni DD, Choi I, Tsukruk VV. Graphene-polymer nanocomposites for structural and functional applications. Prog Polym Sci 2014;39(11):1934-72.

14. J. Huang, C. Mao, Y. Zhu, et al. Control of carbon nanotubes at the interface of a co-continuous immiscible polymer blend to fabricate conductive composites with ultralow percolation thresholds Carbon, 73 (2014), pp. 267-274.

15. Megariotis, G.; Vogiatzis, G.G.; Sgouros, A.P.; Theodorou, D.N. Slip Spring-Based Mesoscopic Simulations of Polymer Networks: Methodology and the Corresponding Computational Code. Polymers 2018, $10,1156$.

16. Vaia RA, Giannelis EP. Lattice model of polymer melt intercalation in organically-modified layered silicates.Macromolecules 1997;30:7990-9.

17. Kim Y, White JL. Modeling of polymer/clay nanocomposite formation. J Appl Polym Sci 2006;101:1657-63.

18. Smith JS, Bedrov D, Smith GD. A molecular dynamics simulation study of nanoparticle interactions in a model polymernanoparticle composite. Compos Sci Technol 2003;63:1599-605.

19. Balazs AC, Singh C, Zhulina E, Lyatskaya Y. Modeling the phase behavior of polymer/clay nanocomposites. Acc. Chem Res 1999;32:651-7.

20. Balazs AC, Singh C, Zhulina E. Modeling the interactions between polymers and clay surfaces through self-consistent field theory. Macromolecules 1998;31:8370-81.

21. Zhulina E, Singh C, Balazs AC. The attraction between surfaces in a polymer melt containing telechelic chains: guidelines for controlling the surface separation in intercalated polymer-clay composites. Langmuir 1999;15:3935-43.

22. Scheutjens J, Fleer GJ. Statistical theory of the adsorption of interacting chain molecules. 2. Train, loop, and tail size distribution. J Phys Chem 1980;84:178-90.

23. Scheutjens J, Fleer GJ. Statistical theory of the adsorption of interacting chain molecules. 1. Partition-function, segment density distribution, and adsorption-isotherms. J Phys Chem 1979;83:1619-35.

24. Carignano MA, Szleifer I. Statistical thermodynamic theory of grafted polymeric layers. J Chem Phys 1993;98:5006-18.

25. Groenewold J, Fredrickson GH. Elastic interactions and stability of clay-filled lamellar phases. Eur Phys J E 2001;5:171-82.

26. Tanaka G, Goettler LA. Predicting the binding energy for nylon 6,6/clay nanocomposites by molecular modeling. Polymer 2002;43:541-53.

27. Fermeglia M, Ferrone M, Pricl S. Computer simulation of nylon-6/organoclay nanocomposites: prediction of the binding energy. Fluid Phase Equilib 2003;212:315-29.

28. Goreke MD, Alakent B, Soyer-Uzun S.Comperitive study on factors govering binding mechanisms in polylacticAcid-Hydroxyapatite and polyethylene - Hydroxyapatite System via MD simulation.Langmuir2020;36:1125-1137.

29. J. Liu, Y. Ma, R. Wu, and M. Yu, "Molecular simulation of diffusion-controlled kinetics in stepwise polymerization," Polymer 97, 335-345 (2016).

30. H. Liu, Y.-L. Zhu, Z.-Y. Lu, and F. Müller-Plathe, “A kinetic chain growth algorithm in coarse-grained simulations,” J. Comput. Chem. 37, 2634-2646 (2016).

31. Lee JY, Baljon ARC, Loring RF, Panagiotopoulos AZ. Simulation of polymer melt intercalation in layered nanocomposites. J Chem Phys 1998;109:10321-30.

32. Baljon ARC, Lee JY, Loring RF. Molecular view of polymer flow into a strongly attractive slit. J Chem Phys 1999;111:906872.

33. Vaia RA, Jandt KD, Kramer EJ, Giannelis EP. Kinetics of polymer melt intercalation. Macromolecules 1995;28: 8080-5.

34. Lee JY, Baljon ARC, Loring RF. Spontaneous swelling of layered nanostructures by a polymer melt. J Chem Phys 1999;111:9754-60.

35. Ginzburg VV, Gendelman OV, Manevitch LI. Simple "kink” model of melt intercalation in polymer-clay nanocomposites. Phys Rev Lett 2001;86:5073-5. 
36. Gendelman OV, Manevitch LI, Manevitch OL. Solitonic mechanism of structural transition in polymer-clay nanocomposites. J Chem Phys 2003;119:1066-9.

37. Balazs AC, Ginzburg VV, Qiu F, Peng GW, Jasnow D. Multi-scale model for binary mixtures containing nanoscopic particles. J Phys Chem B 2000;104:3411-22.

38. Ginzburg VV, Peng G, Qiu F, Jasnow D, Balazs AC. Kinetic model of phase separation in binary mixtures with hard mobile impurities. Phys Rev E 1999;60:4352-9.

39. Anderson KL, Sinsawat A, Vaia RA, Farmer BL. Control of silicate nanocomposite morphology in binary fluids: Coarsegrained molecular dynamics simulations. J Polym Sci Part B: Polym Phys 2005;43:1014-24.

40. Moghadam, S.; Saha.; Dalal, I.; Larson, R.G. Slip-Spring and Kink Dynamics Models for Fast Extensional Flow of Entangled Polymeric Fluids. Polymers 2019, 11, 465.

41. Sefiddashti, M.H.N.; Edwards, B.J.; Khomami, B. Individual Molecular Dynamics of an Entangled Polyethylene Melt Undergoing Steady Shear Flow: Steady-State and Transient Dynamics. Polymers 2019, 11, 476.

42. Wagner HD, Vaia RA. Nanocomposites: issues at the interface. Mater Today 2004;7(11):38-42.

43. Vacatello M. Chain dimensions in filled polymers: an intriguing problem. Macromolecules 2002;35:8191-3.

44. Kloczkowski A, Sharaf MA, Mark JE. Computer-simulations on filled elastomeric materials. Chem Eng Sci 1994;49:2889_ 97.

45. Yuan QW, Kloczkowski A, Mark JE, Sharaf MA. Simulations on the reinforcement of poly(dimethylsiloxane) elastomers by randomly distributed filler particles. J Polym Sci Part B: Polym Phys 1996;34:1647-57.

46. Ozmusul MS, Picu RC. Structure of polymers in the vicinity of convex impenetrable surfaces: the athermal case. Polymer 2002;43:4657-65.

47. Picu RC, Ozmusul MS. Structure of linear polymeric chains confined between impenetrable spherical walls. J Chem Phys 2003;118:11239-48.

48. Starr FW, Schroder TB, Glotzer SC. Molecular dynamics simulation of a polymer melt with a nanoscopic particle. Macromolecules 2002;35:4481-92.

49. Vacatello M. Monte Carlo simulations of polymer melts filled with solid nanoparticles. Macromolecules 2001;34: 1946-52.

50. Vacatello M. Phantom chain simulations of polymer- nanofiller systems. Macromolecules 2003;36:3411-6.

51. Vacatello M. Phantom chain simulations of realistically sized polymer-based nanocomposites. Macromol Theory Simul 2006;15:303-10.11

52. Kuppa V, Menakanit S, Krishnamoorti R, Manias E. Simulation insights on the structure of nanoscopically confined poly(ethylene oxide).

53. Manias E, Kuppa V, Yang DK, Zax DB. Relaxation of polymers in 2 nm slit-pores: confinement induced segmental dynamics and suppresion of the glass transition. Colloids Surf, A 2001;187:509-21.

54. Manias E, Subbotin A, Hadziioannou G, Tenbrinke G. Adsorption-desorption kinetics in nanoscopically confined oligomer films under shear. Mol Phys 1995;85:1017-32.

55. Gaudel-Siri A, Brocorens P, Siri D, Gardebien F, Bredas JL, Lazzaroni R. Molecular dynamics study of epsilon-caprolactone intercalated in Wyoming sodium montmorillonite. Langmuir 2003;19:8287-91.

56. Gardebien F, Bredas JL, Lazzaroni R. Molecular dynamics simulations of nanocomposites based on poly(epsiloncaprolactone) grafted on montmorillonite clay. J Phys Chem B 2005;109:12287-96.

57. Sikdar D, Katti DR, Katti KS. A molecular model for epsilon-caprolactam-based intercalated polymer clay nanocomposite: integrating modeling and experiments. Langmuir 2006;22:7738-47.

58. Katti KS, Sikdar D, Katti DR, Ghosh P, Verma D. Molecular interactions in intercalated organically modified clay and claypolycaprolactam nanocomposites: experiments and modeling. Polymer 2006;47:403-14.

59. Zeng QH, Yu AB, Lu GQ, Standish RK. Molecular dynamics simulation of organic-inorganic nanocomposites: layering behavior and interlayer structure of organoclays. Chem Mater 2003;15:4732-8. 
60. Zeng QH, Yu AB, Lu GQ, Standish RK. Molecular dynamics simulation of the structural and dynamic properties of dioctadecyldimethyl ammoniums in organoclays. J Phys Chem B 2004;108:10025-33.

61. Paul DR, Zeng QH, Yu AB, Lu GQ. The interlayer swelling and molecular packing in organoclays. J Colloid Interface Sci 2005;292:462-8.

62. Zeng QH, Yu AB, Lu GQ, Standish RK. Interfacial interactions and structure of organic-inorganic nanohybrids. J Mater Sci Technol 2005;21:114-8.

63. Greenwell HC, Harvey MJ, Boulet P, Bowden AA, Coveney PV, Whiting A. Interlayer structure and bonding in nonswelling primary amine intercalated clays. Macromolecules 2005;38:6189-200.

64. Heinz H, Vaia RA, Krishnamoorti R, Farmer BL. Selfassembly of alkylammonium chains on montmorillonite: effect of chain length, head group structure, and cation exchange capacity. Chem Mater 2007;19:59-68.

65. Sides SW, Kim BJ, Kramer EJ, Fredrickson GH. Hybrid particle-field simulations of polymer nanocomposites. Phys Rev Lett 2006;96:250601.

66. Wang, W.; Wang, J.; Zhao, Y.; Bai, H.; Huang, M.; Zhang, T.; Song, S. High-performance two-dimensional montmorillonite supported-poly(acrylamide-co-acrylic acid) hydrogel for dye removal. Environ. Pollut. 2020, 257, 113574.

67. Li X, Sun M, Shan C, Chen Q, Wei X. Mechanical properties of 2D materials studied by in situ microscopy techniques. Adv Mater Interfaces 2018;5(5).

68. Roy S, Ryan J, Webster S, Nepal D. A review of in situ mechanical characterization of polymer nanocomposites: prospect and challenges. Appl Mech Rev 2017;69(5): 050802.

69. Subbotin A, Semenov A, Hadziioannou G, Tenbrinke G. Rheology of confined polymer melts under shear flow-weak adsorption limit. Macromolecules 1995;28:3901-3.

70. Subbotin A, Semenov A, Hadziioannou G, tenBrinke G. Nonlinear rheology of confined polymer melts under oscillatory flow. Macromolecules 1996;29:1296-304.

71. Boek ES, Coveney PV, Lekkerkerker HNW, van der Schoot P. Simulating the rheology of dense colloidal suspensions using dissipative particle dynamics. Phys Rev E 1997;55:3124-33.

72. Huh J, Balazs AC. Behavior of confined telechelic chains under shear. J Chem Phys 2000;113:2025-31.

73. Ruths M, Granick S. Tribology of confined Fomblin-Z perfluoropolyalkyl ethers: role of chain-end chemical functionality. J Phys Chem B 1999;103:8711-21.

74. Ruths M, Granick S. Tribology of confined Fomblin-Z perfluoropolyalkylethers: molecular weight dependence and comparison between unfunctionalized and telechelic chains. Tribol Lett 1999;7:161-72.

75. Krishnamoorti R, Ren JX, Silva AS. Shear response of layered silicate nanocomposites. J Chem Phys 2001;114: 4968-73.

76. Smith GD, Bedrov D, Li LW, Byutner O. A molecular dynamics simulation study of the viscoelastic properties of polymer nanocomposites. J Chem Phys 2002;117:9478-89.

77. Starr FW, Douglas JF, Glotzer SC. Origin of particle clustering in a simulated polymer nanocomposite and its impact on rheology. J Chem Phys 2003;119:1777-88.

78. Pryamitsyn V, Ganesan V. Mechanisms of steady-shear rheology in polymernanoparticle composites. J Rheol 2006;50:65583.

79. Sarvestani AS, Picu CR. Network model for the viscoelastic behavior of polymer nanocomposites. Polymer 2004;45: 777990.

80. Song YS, Youn JR. Modeling of rheological behavior of nanocomposites by Brownian dynamics simulation. Korea- Aust Rheol J 2004;16:201-12.

81. Wang YR, Xu JH, Bechtel SE, Koelling KW. Melt shear rheology of carbon nanofiber/polystyrene composites. Rheol Acta 2006;45:919-41.

82. Ghoniem NM, Busso EP, Kioussis N, Huang HC. Multiscale modelling of nanomechanics and micromechanics: an overview. Philos Mag 2003;83:3475-528. 
83. Brechet Y, Cavaille JYY, Chabert E, Chazeau L, Dendievel R, Flandin L, et al. Polymer based nanocomposites: effect of filler-filler and filler-matrix interactions. Adv Eng Mater 2001;3:571-7.

84. Dai ZH, Liu L, Qi X, Kuang J, Wei YG, Zhu HW, Zhang Z. Three-dimensional sponges with super mechanical stability: harnessing true elasticity of individual carbon nanotubes in macroscopic architectures. Sci Rep 2016;6:18930.

85. Roy S, Ryan J, Webster S, Nepal D. A review of in situ mechanical characterization of polymer nanocomposites: prospect and challenges. Appl Mech Rev 2017;69(5): 050802.

86. Dai Z, Wang G, Liu L, Hou Y, Wei Y, Zhang Z. Mechanical behavior and properties of hydrogen bonded graphene/polymer nano-interfaces. Compos Sci Technol 2016;136:1-9.

87. Wang G, Dai Z, Liu L, Hu H, Dai Q, Zhang Z. Tuning the interfacial mechanical behaviors of monolayer graphene/PMMA nanocomposites. ACS Appl Mater Interfaces2016;8(34):22554-62.

88. Xu C, Xue T, Qiu W, Kang Y. Size effect of the interfacial mechanical behavior of graphene on a stretchable substrate. ACS Appl Mater Interfaces 2016;8(40):27099-106.

89. Krishnamoorti R, Vaia RA, Giannelis EP. Structure and dynamics of polymer-layered silicate nanocomposites. Chem Mater 1996;8:1728-34.

90. Qian D, Liu WK, Ruoff RS. Load transfer mechanism in carbon nanotube ropes. Compos Sci Technol 2003;63:1561-9.

91. Gou JH, Minaie B, Wang B, Liang ZY, Zhang C. Computational and experimental study of interfacial bonding of singlewalled nanotube reinforced composites. Comput Mater Sci 2004;31:225-36.

92. Gou JH, Liang ZY, Zhang C, Wang B. Computational analysis of effect of single-walled carbon nanotube rope on molecular interaction and load transfer of nanocomposites. Compos Part B: Eng 2005;36:524-33.

93. Mokashi VV, Qian D, Liu YJ. A study on the tensile response and fracture in carbon nanotube-based composites using molecular mechanics. Compos Sci Technol 2007;67:530-40.

94. Lordi V, Yao N. Molecular mechanics of binding in carbon-nanotube-polymer composites. J Mater Res 2000;15:2770-9.

95. Prathab B, Subramanian V, Aminabhavi TM. Molecular dynamics simulations to investigate polymer-polymer and polymermetal oxide interactions. Polymer 2007;48: 409-16.

96. Aleperstein D, Artzi N, Siegmann A, Narkis M. Experimental and computational investigation of EVOH/clay nanocomposites. J Appl Polym Sci 2005;97:2060-6.

97. Song M, Chen L. Molecular dynamics simulation of the fracture in polymer-exfoliated layered silicate nanocomposites. Macromol Theory Simul 2006;15:238-45.

98. Yoon PJ, Fornes TD, Paul DR. Thermal expansion behavior of nylon 6 nanocomposites. Polymer 2002;43:m6727-41.

99. Zheng XY, Forest MG, Lipton R, Zhou RH. Nematic polymer mechanics: flow-induced anisotropy. Continuum Mech Therm 2007;18:377-94.

100. Brune DA, Bicerano J. Micromechanics of nanocomposites: comparison of tensile and compressive elastic moduli, and prediction of effects of incomplete exfoliation and imperfect alignment on modulus. Polymer 2002;43:369-87.

101. Guz AN, Rodger AA, Guz IA. Developing a compressive failure theory for nanocomposites. Int Appl Mech 2005;41:23355.

102. Raos G, Moreno M, Elli S. Computational experiments on filled rubber viscoelasticity: what is the role of particle-particle interactions. Macromolecules 2006;39: 6744-51.

103. Buxton GA, Balazs AC. Lattice spring model of filled polymers and nanocomposites. J Chem Phys 2002;117: 7649-58.

104. Liu H, Brinson LC. A hybrid numerical-analytical method for modeling the viscoelastic properties of polymer nanocomposites. J Appl Mech 2006;73:758-68.

105. Hbaieb K, Wang QX, Chia YHJ, Cotterell B. Modelling stiffness of polymer/clay nanocomposites. Polymer 2007; 48:9019.

106. Zare Y, Rhee KY. Modelling the effect of filler network and interfacial shear strength on the Mechanical properties of Carbon nanotubes-reinforced nanocomposites.JOM 72(6);2184-2190,2020. 
107. Zare Y,Rhee KY. Effects of interphase regions and filler networks on the viscosity of PLA/PEO/carbon nanotubes Biosensor.

108. Zare Y,Rhee KY. A simulation work for the influence of aggregation/agglomeration of clay layers on the tensile properties of nanocomposites. JOM71(11),3989-3995,2019.

109. Kröger M.Developments in polymer theory and simulation.polymers2020;12,30.

110. Gooneie, A.; Schuschnigg, S.; Holzer, C. A Review of Multiscale Computational Methods in Polymeric Materials. Polymers 2017, 9, 16.

111. Ramirez-Hernandez, A.; Hur, S.M.; Armas-Perez, J.C.; de la Cruz, M.O.; de Pablo, J.J. Demixing by a Nematic Mean Field: Coarse-Grained Simulations of Liquid Crystalline Polymers. Polymers 2017, 9, 88.

112. Megariotis, G.; Vogiatzis, G.G.; Sgouros, A.P.; Theodorou, D.N. Slip Spring-Based Mesoscopic Simulations of Polymer Networks: Methodology and the Corresponding Computational Code. Polymers 2018, 10, 1156.

113. Selytina NS, Borodin EN, Petrov YV.Structural-temporal peculiarities of dynamics eformation of nanostructured and nanoscaled metals.Physics of the solid state60(9),1813-1820,2018.

114. Moghadam, S.; Saha.; Dalal, I.; Larson, R.G. Slip-Spring and Kink Dynamics Models for Fast Extensional Flow of Entangled Polymeric Fluids. Polymers 2019, 11, 465.

115. Ortiz M, Cuitino AM, Knap J, Koslowski M. Mixed atomistic continuum models of material behavior: the art of transcending atomistics and informing continua. MRS Bull 2001;26:216-21. 
